\title{
Compensation of Wave-Induced Motion and Force Phenomena for Ship-Based High Performance Robotic and Human Amplifying Systems
}

October 2003

Prepared by Lonnie J. Love John F. Jansen Francois G. Pin 


\section{DOCUMENT AVAILABILITY}

Reports produced after January 1, 1996, are generally available free via the U.S. Department of Energy (DOE) Information Bridge:

Web site: http://www.osti.gov/bridge

Reports produced before January 1, 1996, may be purchased by members of the public from the following source:

National Technical Information Service

5285 Port Royal Road

Springfield, VA 22161

Telephone: 703-605-6000 (1-800-553-6847)

TDD: $703-487-4639$

Fax: 703-605-6900

E-mail: info@ntis.fedworld.gov

Web site: http://www.ntis.gov/support/ordernowabout.htm

Reports are available to DOE employees, DOE contractors, Energy Technology Data Exchange (ETDE) representatives, and International Nuclear Information System (INIS) representatives from the following source:

Office of Scientific and Technical Information

P.O. Box 62

Oak Ridge, TN 37831

Telephone: $865-576-8401$

Fax: 865-576-5728

E-mail: reports@adonis.osti.gov

Web site: http://www.osti.gov/contact.html

This report was prepared as an account of work sponsored by an agency of the United States Government. Neither the United States government nor any agency thereof, nor any of their employees, makes any warranty, express or implied, or assumes any legal liability or responsibility for the accuracy, completeness, or usefulness of any information, apparatus, product, or process disclosed, or represents that its use would not infringe privately owned rights. Reference herein to any specific commercial product, process, or service by trade name, trademark, manufacturer, or otherwise, does not necessarily constitute or imply its endorsement, recommendation, or favoring by the United States Government or any agency thereof. The views and opinions of authors expressed herein do not necessarily state or reflect those of the United States Government or any agency thereof. 
Compensation of Wave-Induced Motion and Force Phenomena for Ship-Based High Performance Robotic and Human Amplifying Systems

\title{
Lonnie J. Love \\ John F. Jansen \\ Francois G. Pin
}

Date Published: October 2003

\author{
Prepared by \\ OAK RIDGE NATIONAL LABORATORY \\ P.O. Box 2008 \\ Oak Ridge, Tennessee 37831-6285 \\ managed by \\ UT-Battelle, LLC \\ for the \\ U.S. DEPARTMENT OF ENERGY \\ under contract DE-AC05-00OR22725
}




\section{CONTENTS}

Page

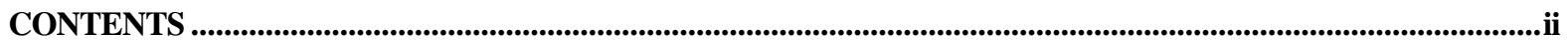

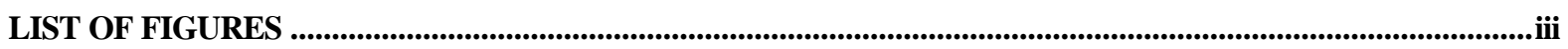

LIST OF TABLES ........................................................................................................................................................

ACKNOWLEDGMENTS ................................................................................................................................... vi

EXECUTIVE SUMMARY........................................................................................................................ vii

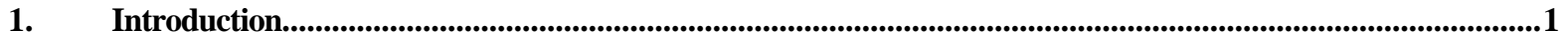

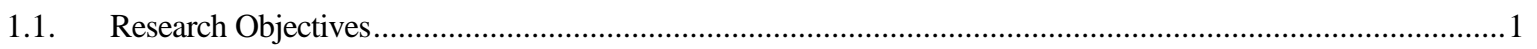

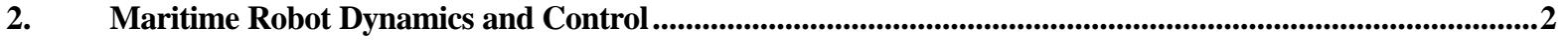

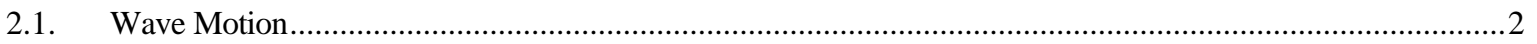

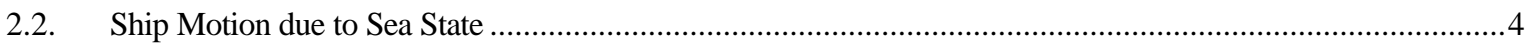

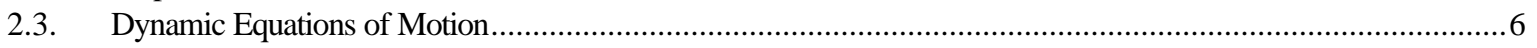

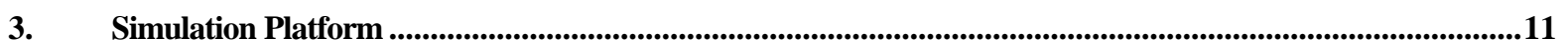

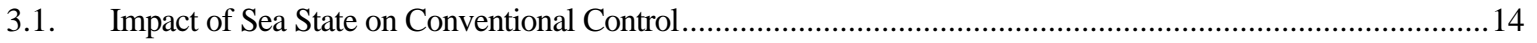

3.2. Preliminary Experiments with Repetitive Learning Control............................................................ 15

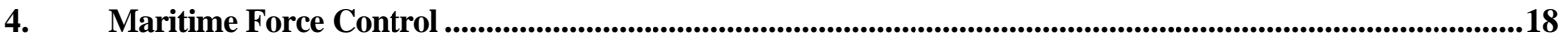

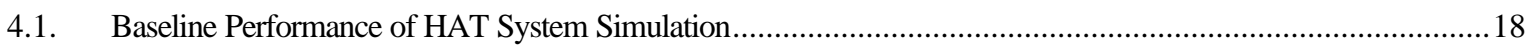

4.2. Ship Motion Compensation for Force Control Strategy ..................................................................23

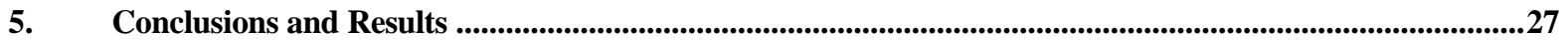

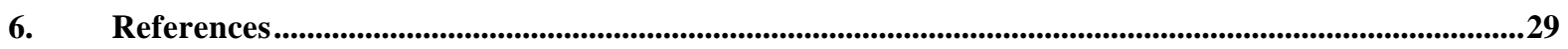

Appendix A : Symbolic Computation of 1 DOF System, 3 Sea States...................................................................

Appendix B : Symbolic Computation of 3 DOF System, Full Sea State .......................................................................

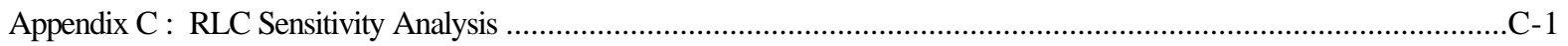

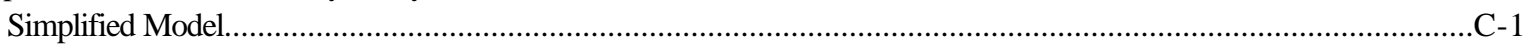

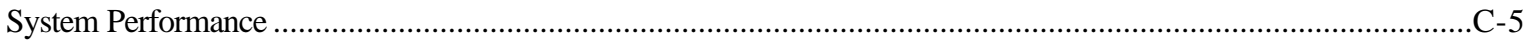




\section{LIST OF FIGURES}

Figure

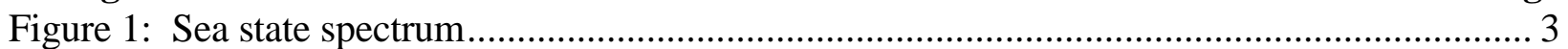

Figure 2: Wave fronts ............................................................................................ 4

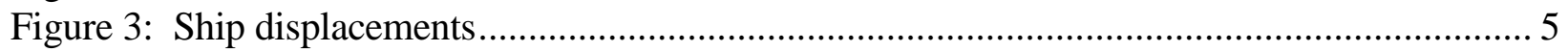

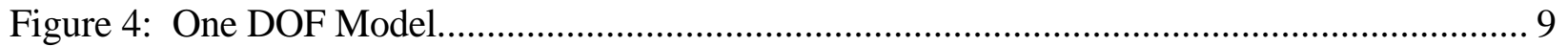

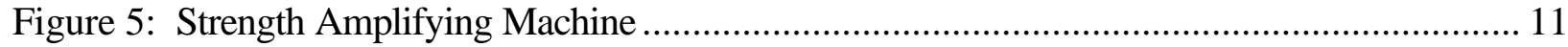

Figure 6: HAT Simulation Model............................................................................. 12

Figure 7: Details of HAT Controller and Manipulator Model................................................ 12

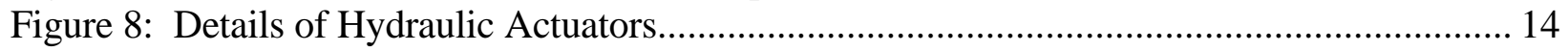

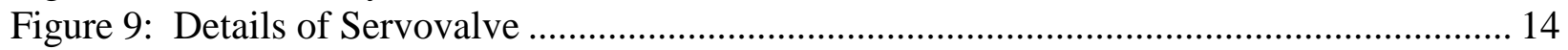

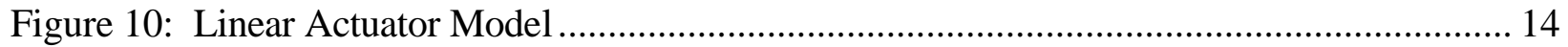

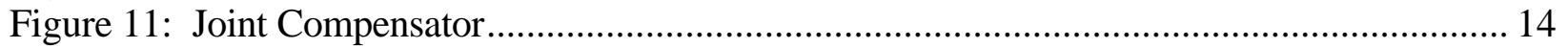

Figure 12: Human Force w/out Compensation.................................................................... 15

Figure 13: Tip Force w/out Compensation..................................................................... 15

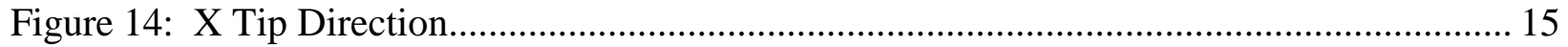

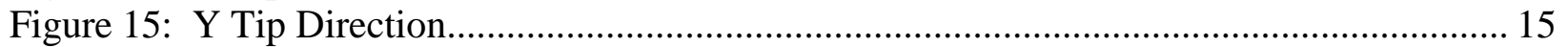

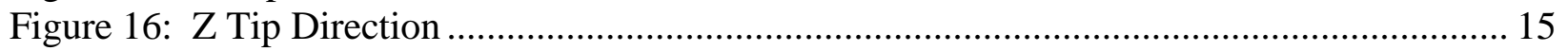

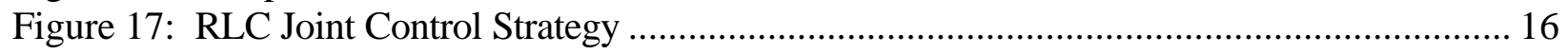

Figure 18: X-Position, simple waves, no compensation..................................................... 16

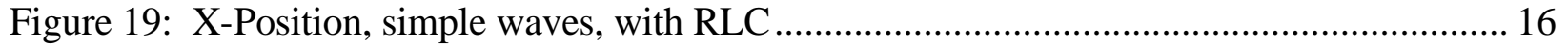

Figure 20: Y-Position, simple waves, no compensation........................................................ 16

Figure 21: Y-Position, simple waves, with RLC ................................................................ 16

Figure 22: Z-Position, simple waves, no compensation ...................................................... 17

Figure 23: Z-Position, simple waves, with RLC ........................................................... 17

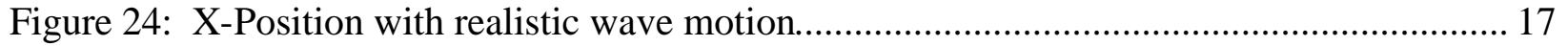

Figure 25: X-Position with RLC and realistic wave motion............................................... 17

Figure 26: Y-Position with realistic wave motion.............................................................. 17

Figure 27: Y-Position with RLC and realistic wave motion................................................ 17

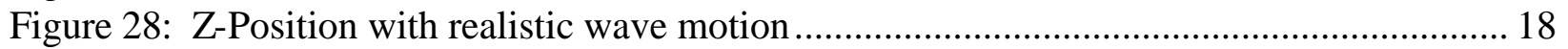

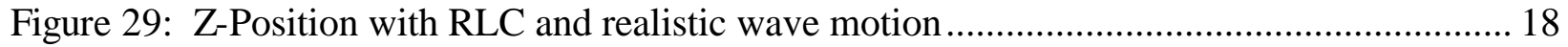

Figure 30: Explicit Force Control ..................................................................................... 20

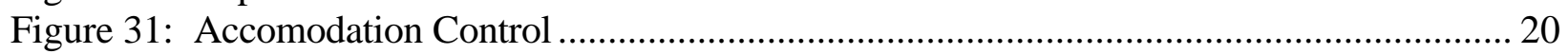

Figure 32: Acceleration Control........................................................................................ 20

Figure 33: ORNL's Next Generation Munitions Handler .......................................................... 20

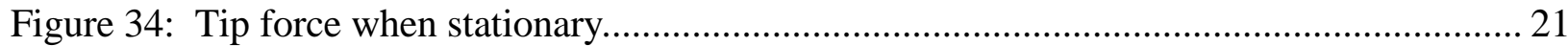

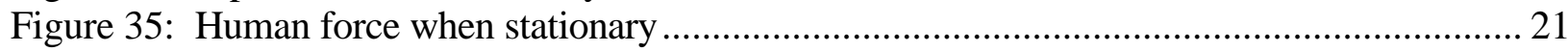

Figure 36: Tip Response to X-Direction Command ....................................................... 22

Figure 37: X-Direction Human Command ........................................................................ 22

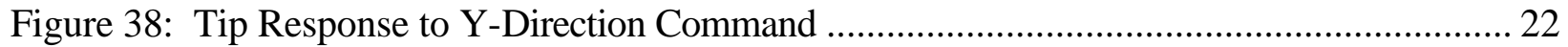

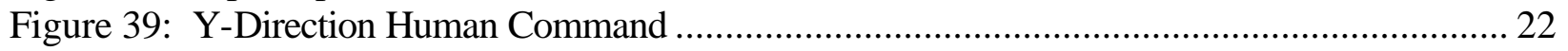

Figure 40: Tip Response to Z-Direction Command ................................................................. 22

Figure 41: Z-Direction Human Command ........................................................................ 22

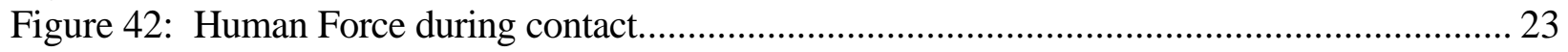




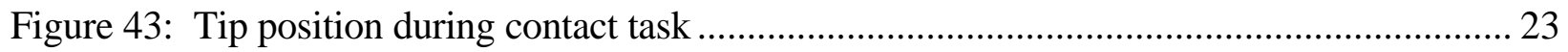

Figure 44: Human Forces at Sea State 5, No Compensation..................................................... 24

Figure 45: Tip Force at Sea State 5, No Compensation.......................................................... 24

Figure 46: X-Position, No Compensation........................................................................ 24

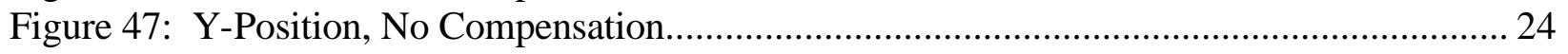

Figure 48: Z-Position, No Compensation ............................................................................. 24

Figure 49: Human Forces at Sea State 5, SMCFCS ............................................................. 26

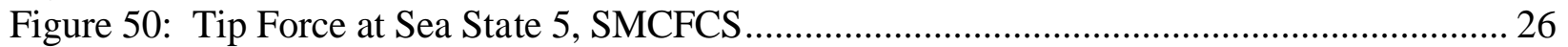

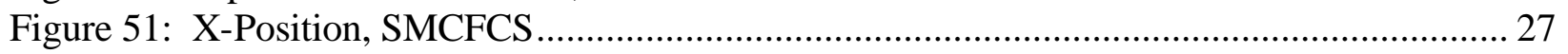

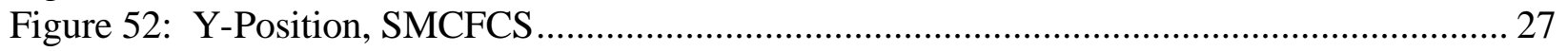

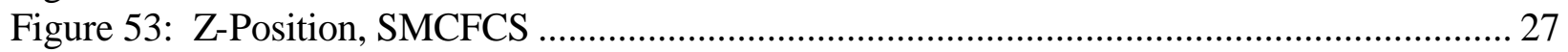

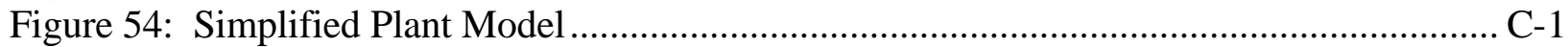

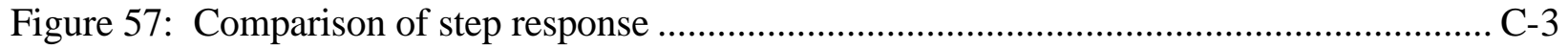

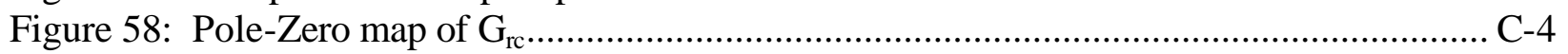

Figure 59: Comparison of Magnitude Response ........................................................... C-5

Figure 60: Comparison of Phase Response …...................................................................... C-5

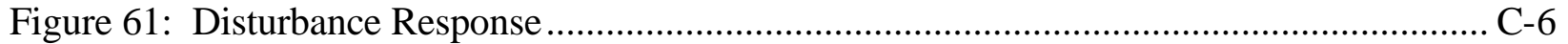

Figure 62: Closed Loop Tracking Performance................................................................ C-6

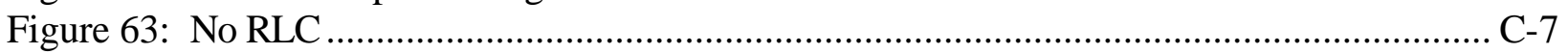

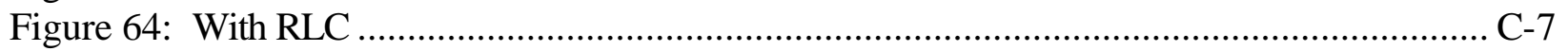

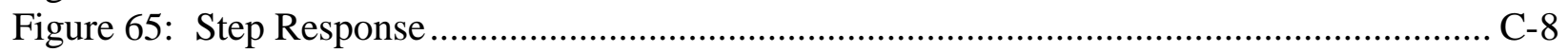

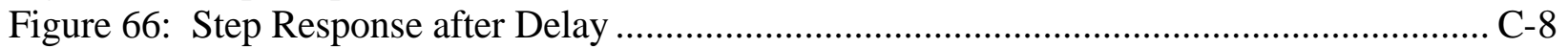




\section{LIST OF TABLES}

Table

Page

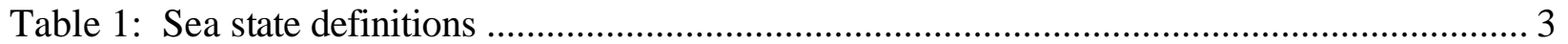

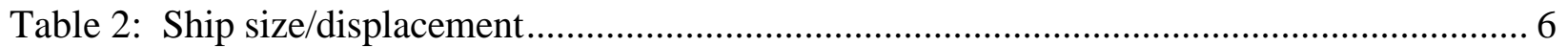

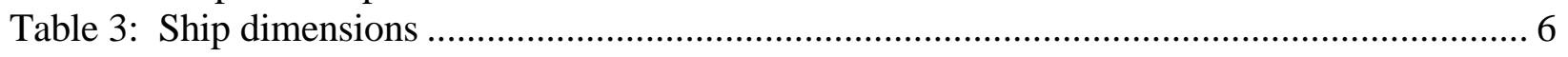

Table 4: Control Performance Comparison.................................................................... 24 


\section{ACKNOWLEDGMENTS}

The authors would like to acknowledge Dr. Theresa McMullen, Mr. Rob Evans, and Ms. Lynn Torres at the Office of Naval Research under Interagency Agreement No. 1866-Q356-A1, whose support made this work possible. 


\section{EXECUTIVE SUMMARY}

The decrease in manpower and increase in material handling needs on many Naval vessels provides the motivation to explore the modeling and control of Naval robotic and robotic assistive devices. This report addresses the design, modeling, control and analysis of position and force controlled robotic systems operating on the deck of a moving ship. First we provide background information that quantifies the motion of the ship, both in terms of frequency and amplitude. We then formulate the motion of the ship in terms of homogeneous transforms. This transformation provides a link between the motion of the ship and the base of a manipulator. We model the kinematics of a manipulator as a serial extension of the ship motion. We then show how to use these transforms to formulate the kinetic and potential energy of a general, multi-degree of freedom manipulator moving on a ship. As a demonstration, we consider two examples: a one degree-of-freedom system experiencing three sea states operating in a plane to verify the methodology and a 3 degree of freedom system experiencing all six degrees of ship motion to illustrate the ease of computation and complexity of the solution. The first series of simulations explore the impact wave motion has on tracking performance of a position controlled robot. We provide a preliminary comparison between conventional linear control and Repetitive Learning Control (RLC) and show how fixed time delay RLC breaks down due to the varying nature wave disturbance frequency. Next, we explore the impact wave motion disturbances have on Human Amplification Technology (HAT). We begin with a description of the traditional HAT control methodology. Simulations show that the motion of the base of the robot, due to ship motion, generates disturbances forces reflected to the operator that significantly degrade the positioning accuracy and resolution at higher sea states. As with position-controlled manipulators, augmenting the control with a Repetitive Learning Controller has little impact due to the variable nature of the wave period. We then introduce a new approach to HAT control, Ship Motion

Compensation for Force Control Systems (SMCFCS). This basic approach uses inclinometer and acceleration information from the base of the robot to compensate for ship motion disturbances. Results of the simulation study show over an order of magnitude decrease in the disturbance force reflected back to the operator and an order of magnitude increase in positioning accuracy and resolution. 


\section{INTRODUCTION}

There is a growing need for robotics in the Navy. Reduction in personnel with no tolerance for reduction in capabilities requires increased levels of both machine automa tion as well as advanced human assistive devices. One example was the development of the Next Generation Munitions Handler (NGMH) by ORNL for the armed services [1]. Traditionally, bomb-loading crews consist of anywhere from four to eight personnel to load bombs and missiles ranging from 500 to 2000 pounds. The current technology to assist crews is based on crude machines with names such as the "jammer" and "hernia bar". With existing and projected future reduction in military workforce, the armed services are exploring the development of technologies that enable fewer personnel to accomplish the same tasks in the same amount of time. There are a wide variety of potential applications of robotic and human assistive systems on a ship (munitions handling, maintenance, damage control, material handling to name a few). However, the nature of the environment provides a host of unique problems. Specifically, the environment in which the robot operates is continually moving. The motion of the ship generates low frequency disturbance forces on the system, both in terms of inertial forces as well as shifts in the direction of gravity. Subsequently, there is a need for the development of advanced control methodologies to compensate for sea state disturbances. While there has been ample research directed towards the design and control of surface vessels, underwater manipulators and vehicles, there has been surprisingly little effort devoted toward the design and control of robotic manipulation systems operating on a ship experiencing heavy sea states. A recent exception is crane control on ships $[2,3,4,5]$. The general problem addressed with maritime crane control is that wave induced motion of a ship produces a low frequency disturbance on the motion of a crane. A robotic system, operating under motion or force control on a ship, will likewise experience low frequency disturbances that can impact the precision and performance of the machine. This report addresses two fundamental problems associated with maritime robotics. First, the nature of the environment makes it difficult to experimentally test competing control methodologies in a laboratory setting. There are only a handful of sea state simulation platforms that have the capacity to hold a moderately sized robotic system. [6,7,8] However, much can be gained by having a high fidelity numerical simulation of a robot that includes ship motion disturbances. The first portion of this report focuses on a high fidelity simulation capability for general manipulation systems operating on ships in heavy sea states. The remaining effort is devoted to an exploration of control methodologies devoted to masking sea state disturbances on maritime human strength amplification systems.

\subsection{Research Objectives}

The focus of this research was the exploration of fundamental control concepts for wave motion-induced disturbance compensation on general manipulation systems. This includes robotic, teleoperated, and human assist devices that are, and will increasingly be, used on-board ships. Both the frequency and amplitude of the disturbances generated on ship-based systems by wave motions are typically variable, either as a direct result of changes in the sea motion, or as the result of movement of the system to different locations on the ship. Existing compensation control methodologies are limited in scope and capabilities to fixed frequency disturbances on systems with known dynamic characteristics. Our objective was to develop novel concepts and methodologies for compensation control of disturbances that can vary in frequency as well as amplitude and are applicable to a very broad class of systems including highly nonlinear time invariant systems. 
One pervasive problem associated with control design for maritime systems is experimental validation of the control performance. While there are many existing sea state systems (Naval ships), experimental validation of control design on sea faring ships is prohibitively difficult due to cost, time, and availability. There are existing land-based sea state simulators, however there are still similar difficulties in terms of access and flexibility. Subsequently, one of the preliminary objectives of this research is to formulate a simple and efficient methodology to derive the dynamic equations of motion for a multi-degree of freedom manipulator moving on a six degree of freedom platform with emphasis placed on numerical simulation. The motivation is to develop a basic framework for developing a high fidelity numerical simulation. We begin in Section II with a basic description of models used to describe a variety of sea states. This is followed by an abbreviated analysis of the motion of a marine vessel experiencing wave loading. The results of this section provide some relevant information quantifying the amplitude and frequency of disturbances expected for a variety of vessels operating under various sea states. We show how the classic homogeneous transform, combined with the energy approach can easily be configured to symbolically calculate the dynamic equations of motion of a general serial link manipulator operating on the deck of a moving ship. We model the ship motion as a six-degree of freedom system. The manipulation system is coupled serially to the ship model. Elements of the homogeneous transform can be used to symbolically compute the position, velocity, angular position, and angular velocity vectors of the center of mass of each link and the payload of the manipulator. This basic methodology is applicable to any symbolic computation program. However, we use Matlab ${ }^{\circledR}$ and the Symbolic Toolbox and show through two examples how the resulting symbolic equations of motion can be easily integrated with Simulink ${ }^{\circledR}$ to provide a numerical simulation of the system. In section III, we exploit this simulation capability to explore position and force control issues of a general maritime manipulation system. We begin with a detailed description of one example system: an existing three degree of freedom human amplification system. The simulation model includes detailed modeling of the nonlinear dynamics of both the arm and hydraulic actuation system. As a preliminary example, we explore the impact sea state disturbances have on the accuracy of the system under position control. We then explore the potential for using Repetitive Learning Control (RLC) to suppress the impact of periodic sea state disturbances. In Section IV, emphasis shifts towards force control, specifically human amplification technology. We provide a detailed description of the basic methodology and use the simulation tool to demonstrate the basic characteristics of the system as well as the impact of sea motion. As with position-controlled systems, we show how RLC is limited to only periodic disturbances and breaks down with realistic waves. However, we then introduce a simple sensory-based approach to suppress sea state disturbances on a force controlled system. Simulation results show an order of magnitude reduction in the disturbance forces reflected to the operator and an order of magnitude reduction in the tracking error. The motivation for this work is to develop a platform for testing control algorithms and alternative designs for ship-board robotic and human assistive machines.

\section{MARITIME ROBOT DYNAMICS AND CONTROL}

\subsection{Wave Motion}

We begin by considering the source of our problem, wave motion. There has been considerable effort devoted towards the modeling and analysis of wave motion. An irregular wave pattern can be generated through a combination of sinusoidal waves of different amplitudes and frequencies. Since standard waves 
are characterized as a combination of wave amplitudes and frequencies, it is standard practice to model wave motion as an energy spectrum. The actual units of the spectral model are normalized with respect to water density and gravity, thus the units are in terms of displacement squared over frequency. One popular model is the two-parameter Bretschneider wave spectral model used to define the frequency content of random sea waves. The two parameters are, by definition, the significant wave height $\left(\mathrm{H}_{1 / 3}\right)$ in centimeters, and the modal wave period (T) in seconds. This significant wave height is defined as the average height of the top 1/3 highest waves. The wave spectral density, $\mathrm{S}\left(\omega_{\mathrm{w}}\right)$, is defined in Equation (1).

$$
\mathrm{S}(\omega)=\frac{\mathrm{A}}{\omega^{5}} \mathrm{e}^{-\mathrm{B} / \omega^{4}} \text { with } \mathrm{A}=173 \mathrm{H}_{1 / 3}^{2} / \mathrm{T}^{4} \text { and } \mathrm{B}=691 / \mathrm{T}^{4}
$$

It is common to describe the wave conditions, which include $\mathrm{H}_{1 / 3}$ and $\mathrm{T}$, in terms of a specific "sea state". Table I provides a condensed version of the relevant data from the popular Pierson-Moskowitz sea condition definition [9]. Figure 1 shows a representative spectrum for sea state 5.

Table 1: Sea state definitions

\begin{tabular}{|l|l|l|l|l|}
\hline $\begin{array}{l}\text { Sea } \\
\text { State }\end{array}$ & Description & $\begin{array}{l}\text { Wind velocity } \\
\text { (knots) }\end{array}$ & $\begin{array}{l}\text { Significant } \\
\text { height (ft) }\end{array}$ & $\begin{array}{l}\text { wave } \\
\text { Average } \\
\text { period (sec) }\end{array}$ \\
\hline 0 & Ripples with appearance of scales, without foam crests & $0-2$ & $0-0.01$ \\
\hline 1 & Small wavelets, short but pronounced crests do not break & $5-8.5$ & 0.5 \\
\hline 2 & Small waves becoming larger & $10-13.5$ & $0.5-1.3$ & $1.3-3.3$ \\
\hline 3 & Fairly frequent "white horses" & $14-16$ & $3.6-4.7$ & $2.7-3.6$ \\
\hline 4 & Moderates waves, taking a more pronounced long form. & $18-20$ & $3.8-4.3$ & $4.8-5.1$ \\
\hline 5 & Large waves begin to form. & $22-24$ & $7.3-10.5$ & $5.4-6.4$ \\
\hline 6 & White crests are more extensive everywhere. & $25-28$ & $10.9-14.3$ \\
\hline 7 & $\begin{array}{l}\text { Sea heaps up and white foam from braking waves blown in } \\
\text { streaks along wind direction }\end{array}$ & $30-40$ & $16.4-29.1$ \\
\hline 8 & High waves. Sea begins to roll. Visibility affectes. & $42-54$ & $8.6-7.5$ \\
\hline 9 & Rolling of the sea becomes heavy and shocklike. & $>54$ & $40.8-67.4$ \\
\hline
\end{tabular}

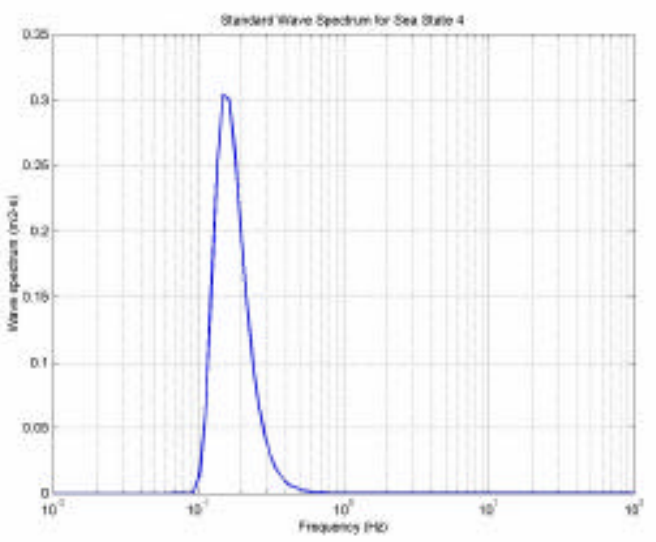

Figure 1: Sea state spectrum

The distribution of wave spectral energy as a function of ship heading is considered either to be unidirectional (long crested) or spread $\pm 90^{\circ}$ about a predominant direction (short crested), shown in Figure 2. Equation (2) accounts for the spread of the energy, transforming from long crested to short crested wave 
models,

$$
\mathrm{S}(\omega, v)=(2 / \pi) \cos ^{2}(v-\mu) \mathrm{S}(\omega)
$$

where $\mu$ is the predominant heading of the wave front containing the principal amount of energy and a $v$ represents the variation of wave energy as a function of the predominant direction of the wave front. There are a variety of methods available for the computation of the wave amplitude time history. It is common practice to quantize the above energy spectrum into $\mathrm{N}$ equally spaced elements. The amplitude, $\zeta_{\mathrm{k}}$, at the discrete frequency $\omega_{\mathrm{k}}$, is extracted from the spectral energy in Equation (1) and Figure 1. Equation (3) is the discretized expression for long crested waves

$$
\zeta_{L C}(t)=\sum_{k=1}^{N} \zeta_{k} \cos \left(\omega_{E k} t+\gamma_{k}\right)
$$

where $\omega_{\mathrm{Ek}}$ is the encountered wave frequency and $\gamma_{\mathrm{k}}$ is a random phase angle. The encountered wave frequency is actually a doppler shift in the wave frequency, $\omega$, as a function of ship speed $(\mathrm{V})$ and heading $(\mu)$.

$$
\omega_{\mathrm{Ek}}=\left|\omega-\left(\omega^{2} \mathrm{~V} / \mathrm{g}\right) \cos (\mu)\right|
$$

The component amplitude, $\zeta_{\mathrm{k}}$, is computed by:

$$
\zeta_{\mathrm{k}}=\sqrt{2 \int_{\omega_{\mathrm{k}}-\mathrm{d} \omega / 2}^{\omega_{\mathrm{k}}+\mathrm{d} \omega / 2} \mathrm{~S}_{\zeta}(\omega) \mathrm{d} \omega}
$$

The number of frequencies, $\mathrm{N}$, used to compute the wave time history should be large enough to obtain a representative Raleigh distribution of single amplitudes. Likewise, the increment in frequency, d $\omega$, is equal to the range of frequencies $\left(\omega_{\max }-\omega_{\min }\right)$ divided by $\mathrm{N}$ where $\omega_{\max }$ and $\omega_{\min }$ are based upon the frequency range that provides ample representation of the total wave energy. The computation of short crested wave time history is slightly more complex, accounting for angular spread in wave energy. The above description provides the background necessary for the computation of wave time histories.

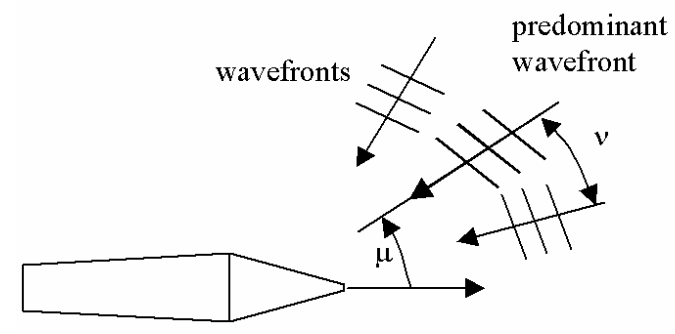

Figure 2: Wave fronts

\subsection{Ship Motion due to Sea State}

There has been a great deal of effort devoted towards modeling ship motion due to wave loadings. However, the primary focus had been directed towards ship design [10,11,12]. Our motivation for understanding ship motion is to quantify the expected magnitude and frequency of disturbance loads for a motion and/or force controlled manipulation system. Subsequently, this section will provide an abbreviated explanation of one of the techniques presently used to model ship motion. 


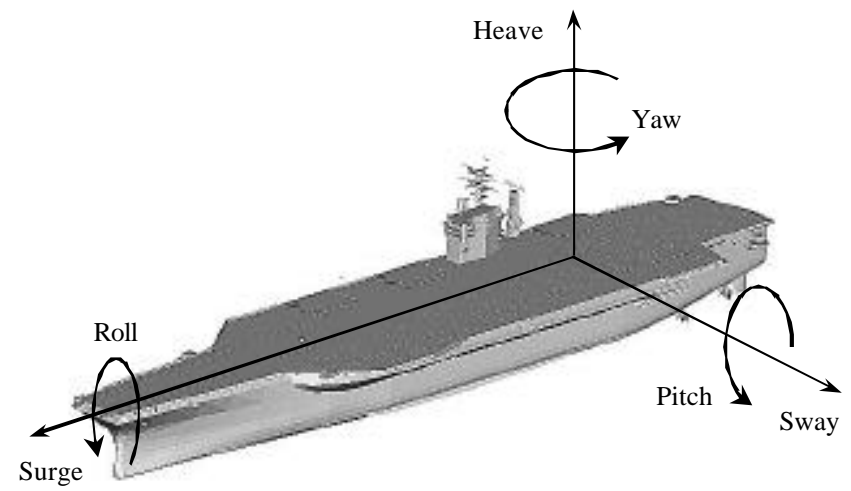

Figure 3: Ship displacements

Figure 3 shows a simplified model of a ship with the corresponding displacements due to wave motion. The motion of the ship is defined by six displacements (surge, sway, heave, roll, pitch, and yaw) at the ship's longitudinal center of gravity, from which motions at all other locations on the ship can be developed. While there are a number of techniques to simulate ship motion, the strip theory of Salvensen et al. is one of the more popular approaches to modeling the 6 DOF response for a ship advancing at a constant forward speed with arbitrary heading in regular sinusoidal waves [13]. In it's simplest form, a ship acts as a set of filters, called the Response Amplitude Operators (RAOs), that transforms wave motion into the six degrees of motion (surge, sway, heave, roll, pitch and yaw). Each degree of freedom has its own characteristic RAO. As illustrated in the previous section, there is ample information for characterizing the frequency content of the waves. The challenge is to design accurate models of the ship that faithfully characterizes the behavior of the ship. Strip theory is able to provide reliable estimates of RAO's for a wide range of hull forms and sea conditions. There are three main stages to computing the motion response of the ship. First, divide the ship into a number of transverse sections (or strips), generally from 10 to 40 , and compute the two-dimensional hydrodynamic coefficients such as added mass, damping, wave excitation and restoring force. Next, integrate these values along the length of the vessel to obtain global coefficients for the coupled motion of the vessel. Finally, the equations of motion for the ship can be solved to give the amplitudes and phases of the heave, surge, sway, yaw, pitch and roll motions. Clearly, the motion of a ship is a complex phenomenon and the above description is merely a simplified explanation of one method used for modeling ship motion. The above description is intended to only provide insight into the problem of ship motion simulation. The interested reader is referred to the following list of articles for a deeper understanding of ship motion simulation $[14,15,16]$. Fortunately, there are a number of commercial software packages available for the analysis and simulation of marine vessels. The level of sophistication, as well as magnitude of cost, varies dramatically. The package used for the analysis in the paper is the Simulation Time History (STH) and Access Time History (ACTH) programs developed at the Naval Surface Warfare Center in Bethesda Maryland and are available through the National Technical Information Service (NTIS). 
Table 2: Ship size/displacement

\begin{tabular}{|c|c|c|c|c|c|c|c|c|c|}
\hline $\begin{array}{c}\text { Sea } \\
\text { State }\end{array}$ & $\begin{array}{c}\text { Wave } \\
\text { Ht } \\
(\mathrm{ft})\end{array}$ & $\begin{array}{c}\text { Bength } \\
(\mathrm{ft})\end{array}$ & $\begin{array}{c}\text { Pitch } \\
(\mathrm{deg})\end{array}$ & $\begin{array}{c}\text { Pitch } \\
\text { Period } \\
(\mathrm{sec})\end{array}$ & $\begin{array}{c}\text { Heave } \\
(\mathrm{ft})\end{array}$ & $\begin{array}{c}\text { Heave Acc } \\
(\mathrm{g})\end{array}$ & $\begin{array}{c}\text { Beam } \\
(\mathrm{ft})\end{array}$ & $\begin{array}{c}\text { Roll } \\
\text { Angle } \\
(\mathrm{deg})\end{array}$ & $\begin{array}{c}\text { Roll } \\
\text { Period } \\
(\mathrm{sec})\end{array}$ \\
\hline 4 & 6.8 & $<150$ & 2 & 3.5 & 2.2 & 0.10 & $<50$ & 7 & 7.1 \\
\hline & & $150-250$ & 2 & 4 & 2.2 & 0.10 & $50-75$ & 6 & 11.5 \\
\hline & & $250-350$ & 1 & 5 & 2.2 & 0.10 & $75-105$ & 6 & 13.7 \\
\hline & & $350-500$ & 1 & 6 & 1.7 & 0.08 & $>105$ & 5 & 14.8 \\
\hline & & $500-700$ & 1 & 7 & 1.3 & 0.06 & & & \\
\hline & & $>700$ & 1 & 8 & 0.9 & 0.04 & & & \\
\hline 5 & 9.8 & $<150$ & 3 & 3.5 & 5.2 & 0.17 & $<50$ & 12 & 7.1 \\
\hline & & $150-250$ & 3 & 4 & 5.2 & 0.17 & $50-75$ & 10 & 11.5 \\
\hline & & $250-350$ & 2 & 5 & 5.2 & 0.17 & $75-105$ & 10 & 13.7 \\
\hline & & $350-500$ & 2 & 6 & 4.3 & 0.14 & $>105$ & 9 & 14.8 \\
\hline & & $500-700$ & 2 & 7 & 3.1 & 0.10 & & & \\
\hline & & $>700$ & 1 & 8 & 2.1 & 0.07 & & & 7.1 \\
\hline 6 & 17 & $<150$ & 5 & 3.5 & 15.0 & 0.27 & $<50$ & 19 & 11.5 \\
\hline & & $150-250$ & 4 & 4 & 15.0 & 0.27 & $50-75$ & 16 & 13.7 \\
\hline & & $250-350$ & 4 & 5 & 15.0 & 0.27 & $75-105$ & 15 & 13 \\
\hline & & $350-500$ & 3 & 6 & 11.7 & 0.21 & $>105$ & 13 & 14.8 \\
\hline & & $500-700$ & 3 & 7 & 8.9 & 0.16 & & & \\
\hline & & $>700$ & 2 & 8 & 6.1 & 0.11 & & & \\
\hline
\end{tabular}

Table 3: Ship dimensions

\begin{tabular}{|l|l|l|l|}
\hline Type of ship & Displacement (tons) & Length (ft) & Beam (ft) \\
\hline Aircraft Carrier & 97,000 & 1092 & 134 \\
\hline Queen Elizabeth II & 66,000 & 887 & 103 \\
\hline Battleship & 59,000 & 860 & 108 \\
\hline Amphibious Assault & 40,500 & 844 & 106 \\
\hline S.S. Rotterdam & 38,650 & 748 & 94 \\
\hline Ammunition ship & 18,000 & 564 & 81 \\
\hline Command ship & 14,650 & 520 & 84 \\
\hline Cruiser, Destroyer & 9600 & 567 & 55 \\
\hline Frigates & 4100 & 445 & 45 \\
\hline Rescue and Salvage & 3280 & 255 & 51 \\
\hline Coastal patrol & 170 & 33 \\
\hline
\end{tabular}

Table 2 provides a general description of the expected motion of a ship based upon the sea state and vessels length and beam dimensions [15]. A listing of naval and commercial vessels with their respective displacement, length and beam dimensions follow this in Table 3. A full listing of the data on each of the above Navy war ships is available through a Navy web site [16].

\subsection{Dynamic Equations of Motion}

At this point, we have the ability to model the motion of a ship as a function of the sea state and vessel. We now are interested in understanding the impact this ship motion has on the dynamics of a general manipulation system. Our approach to modeling the dynamics of a robot on a moving platform, such as a ship, consists of: modeling the ship motion and robot kinematics with homogeneous transforms, constructing kinetic and potential energy terms using these transforms, and symbolically computing the dynamic equations of motion via the Lagrange approach. First, as a review, the homogeneous transform is expressed using the traditional Denavit-Hartenberg (D-H) representation found in most robotics texts where the four quantities $\theta_{\mathrm{i}}$ (angle), $\alpha_{i}$ (twist), $d_{i}$ (offset), $1_{i}$ (length) are parameters of link and joint i [17, 18].

$$
\mathrm{H}_{\mathrm{i}}=\left[\begin{array}{cccc}
\mathrm{c}_{\theta \mathrm{i}} & -\mathrm{s}_{\theta \mathrm{i}} \mathrm{c}_{\alpha \mathrm{i}} & \mathrm{s}_{\theta \mathrm{i}} \mathrm{s}_{\alpha \mathrm{i}} & \mathrm{a}_{\mathrm{i}} \mathrm{c}_{\theta \mathrm{i}} \\
\mathrm{s}_{\theta \mathrm{i}} & \mathrm{c}_{\theta \mathrm{i}} \mathrm{c}_{\alpha \mathrm{i}} & -\mathrm{c}_{\theta \mathrm{i}} \mathrm{s}_{\alpha \mathrm{i}} & \mathrm{a}_{\mathrm{i}} \mathrm{s}_{\theta \mathrm{i}} \\
0 & \mathrm{~s}_{\alpha \mathrm{i}} & \mathrm{c}_{\alpha \mathrm{i}} & \mathrm{d}_{\mathrm{i}} \\
0 & 0 & 0 & 1
\end{array}\right]
$$

The conventional use of the homogeneous transform treats each subsequent transformation as a body 
fixed rotation and translation. However, the sea state is generally described in terms of space fixed displacements. e.g. all of the translations and rotations are with respect to the same space fixed reference frame. Referring to Figure 3, roll and surge are about a fixed X-axis, pitch and sway are about a fixed Yaxis and yaw and heave are about a fixed $\mathrm{Z}$-axis. We begin by constructing a homogeneous transformation using space-fixed rotations and translations for a transformation from the sea coordinate frame to the base of the robot. Equation (7) is the final expression for the displacement of the base of a robot with respect to a sea state where $c_{\theta}$ is $\cos (\theta)$ and $s_{\theta}$ is $\sin (\theta)$.

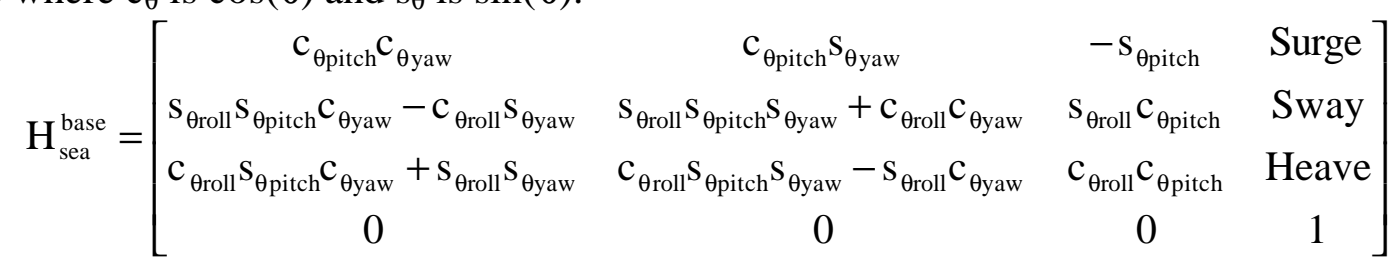

We assume for now that the sea states are defined with respect to the base of our robot. If necessary, additional transformations can be included from the coordinate system of the sea state to the base of the manipulator. We also assume that we can define homogeneous transforms from each joint to a point on each link where the associated mass properties (mass and inertia matrix) are known. So, our basic methodology consists of using the homogenous transforms to identify the displacements and velocities, both translation and rotation, of the center of mass of each link and payload with respect to the manipulators state and the sea state. We extract out of the transforms the vertical displacement of each center of mass for an expression of the total potential energy of the system. Likewise, computation of the system's kinetic energy is based on computing the linear and angular and velocity of each link center of gravity with respect to the inertial frame. Once the kinetic and potential energy terms are derived, we simply use the jacobian() function to symbolically calculate the mass matrix and nonlinear dynamic terms following the Lagrange formulation.

First, the position of the center of mass for each link, with respect to the system's inertial coordinate system, is computed by post multiplying the displacement of the robot base with respect to the sea, $\mathrm{H}_{\text {sea }}^{\text {base }}$, by a homogeneous transform from the robot base to the link's c.g., $\mathrm{H}_{\text {base }}^{\mathrm{i}}$.

$$
\begin{aligned}
& \mathrm{H}_{\text {sea }}^{\mathrm{i}}=\mathrm{H}_{\text {sea }}^{\text {base }} \mathrm{H}_{\text {base }}^{\mathrm{i}} \\
& =\left[\begin{array}{cc}
\mathrm{R}_{\text {sea }}^{\text {base }} & \overline{\mathrm{x}}_{\text {sea }}^{\text {base }} \\
\overline{0} & 1
\end{array}\right]\left[\begin{array}{cc}
\mathrm{R}_{\text {base }}^{\mathrm{i}} & \overline{\mathrm{x}}_{\text {base }}^{\mathrm{i}} \\
\overline{0} & 1
\end{array}\right] \\
& =\left[\begin{array}{cc}
\mathrm{R}_{\text {sea }}^{\mathrm{i}} & \overline{\mathrm{x}}_{\text {sea }}^{\mathrm{i}} \\
\overline{0} & 1
\end{array}\right], \overline{\mathrm{x}}_{\text {sea }}^{\mathrm{i}}=\left\{\begin{array}{c}
\mathrm{x}_{\text {sea }}^{\mathrm{i}} \\
\mathrm{y}_{\text {sea }}^{\mathrm{i}} \\
\mathrm{z}_{\text {sea }}^{\mathrm{i}}
\end{array}\right\}
\end{aligned}
$$

The potential energy due to gravity for link $\mathrm{i}$ is the vertical component ( $\mathrm{z}_{\text {sea }}^{\mathrm{i}}$ in direction of gravity) of $\overline{\mathrm{x}}_{\text {sea }}^{\mathrm{i}}$ times the mass of the link.

$$
\mathrm{V}^{\mathrm{i}}=\mathrm{m}_{\mathrm{i}} \mathrm{gz} \mathrm{z}_{\text {sea }}^{\mathrm{i}}
$$

To compute the kinetic energy, we must first derive expressions for the linear and angular velocity of the center of gravity for each link as a function of the sea state and states of the manipulator. We have an expression, $\bar{x}_{\text {sea }}^{i}$ in Equation (8), for the position of the c.g. of link i with respect to the sea inertial frame. The velocity vector, $\overline{\mathrm{v}}_{\mathrm{i}}$, is computed by multiplying the Jacobian (with respect to the combined states of the manipulator $\overline{\mathrm{q}}_{\text {base }}^{\mathrm{i}}$ and sea displacment $\left.\overline{\mathrm{q}}_{\text {sea }}\right)$ of $\overline{\mathrm{X}}_{\text {sea }}^{\mathrm{i}}, \mathrm{J}\left(\overline{\mathrm{x}}_{\text {sea }}^{\mathrm{i}}, \overline{\mathrm{q}}\right)$, by the state velocity vector, $\dot{\overline{\mathrm{q}}}$. Note from equation (10) that $\overline{\mathrm{q}}_{\text {sea }}$ is a vector of the sea displacement, $\overline{\mathrm{q}}_{\text {base }}^{\mathrm{i}}$ is a vector of robot joint displacements 
from the base of the robot (joint $\left.\mathrm{q}_{0}\right)$ to the $\mathrm{i}^{\text {th }}$ joint $\left(\mathrm{q}_{\mathrm{i}}\right)$. The displacement vector $\overline{\mathrm{q}}$ combines the sea state displacement with the robot joint displacement vector.

$$
\begin{aligned}
& \overline{\mathrm{v}}_{\mathrm{i}}=\frac{\partial \overline{\mathrm{x}}_{\text {sea }}^{\mathrm{i}}}{\partial \mathrm{t}} \\
& =\sum_{\mathrm{j}=1}^{6+\mathrm{i}} \frac{\partial \overline{\mathrm{x}}_{\text {sea }}^{\mathrm{i}}}{\partial \mathrm{q}_{\mathrm{j}}} \dot{\mathrm{q}}_{\mathrm{j}} \\
& =\mathrm{J}\left(\overline{\mathrm{x}}_{\text {sea }}^{\mathrm{i}}, \overline{\mathrm{q}}\right) \dot{\overline{\mathrm{q}}} \\
& \text { where } \overline{\mathrm{q}}=\left[\overline{\mathrm{q}}_{\text {sea }} \overline{\mathrm{q}}^{\mathrm{i}}\right] \\
& \text { with } \overline{\mathrm{q}}_{\text {sea }}=[\text { roll }, \text { pitch, yaw, surge, sway, heave }] \\
& \text { and } \overline{\mathrm{q}}_{\text {base }}^{\mathrm{i}}=\left[\mathrm{q}_{0} \mathrm{q}_{1} \ldots \mathrm{q}_{\mathrm{i}}\right]
\end{aligned}
$$

The rotational velocity is a little more involved, but can be simplified by again using the homogeneous transform. Starting at the base of the robot, project the net rotational velocity vector forward to the c.g. of each link using the rotational component, $\mathrm{R}_{\text {sea }}^{\mathrm{i}}$. We begin by defining the base rotational velocity.

$$
\bar{\omega}^{\text {base }}=\left[\begin{array}{lll}
\dot{\theta}_{\text {roll }} & \dot{\theta}_{\text {pitch }} & \dot{\theta}_{\text {yaw }}
\end{array}\right]
$$

We combine the rotational velocity of the first link (with respect to the link), $\dot{\overline{\mathrm{q}}}_{1}$, with the projection of $\omega^{\text {base }}$ to the center of mass of the link, again using the rotational component of the homogeneous transform in Equation (7).

$$
\bar{\omega}_{1}=\dot{\bar{q}}_{1}+R_{\text {base }}^{1} \bar{\omega}^{\text {base }}
$$

Each subsequent joint consists of projecting the total angular velocity vector of the previous joint to the current joint's coordinate system, using the rotational component of that joint's homogenous transform, and adding the joint angular velocity.

$$
\bar{\omega}_{i}=\dot{\overline{\mathrm{q}}}_{\mathrm{i}}+\mathrm{R}_{\mathrm{i}-1}^{\mathrm{i}} \bar{\omega}_{\mathrm{i}-1}
$$

We now have expressions for the linear and angular velocity of the center of mass for each link. The total kinetic energy of the system is

$$
\mathrm{T}=\frac{1}{2} \sum_{\mathrm{i}=1}^{\mathrm{N}} \mathrm{m}_{\mathrm{i}} \mathrm{v}_{\mathrm{i}}^{\mathrm{t}} \mathrm{v}_{\mathrm{i}}+\mathrm{R}_{\text {base }}^{\mathrm{i}} \bar{\omega}_{\mathrm{i}}^{\mathrm{t}}\left[\mathrm{I}_{\mathrm{i}}\right] \bar{\omega}_{\mathrm{i}}
$$

where $m_{i}$ is the mass of link $i$ and $I_{i}$ is the inertia matrix of link $i$ about the center of gravity. As a final step, we add external forces applied to the system. For now, we assume forces are applied only to the joints and tip of the robot. We use the principle of virtual work to lump these terms together.

$$
\begin{aligned}
& \partial \mathrm{W}=\sum_{\mathrm{i}=1}^{\mathrm{N}} \tau_{\mathrm{i}} \partial \mathrm{q}_{\mathrm{i}}+\overline{\mathrm{F}}_{\text {tip }} \partial \overline{\mathrm{x}}_{\text {tip }}+\overline{\mathrm{M}}_{\text {tip }} \partial \theta_{\text {tip }} \\
& =\left[\bar{\tau}+\mathrm{J}_{\text {tip }}^{\mathrm{t}}(\overline{\mathrm{q}})\left\{\frac{\overline{\mathrm{F}}_{\text {tip }}}{\overline{\mathrm{M}}_{\text {tip }}}\right\}\right] \partial \overline{\mathrm{q}} \\
& =\overline{\mathrm{Q}} \partial \overline{\mathrm{q}} \\
& \mathrm{J}_{\text {tip }}(\overline{\mathrm{q}})=\frac{\partial \overline{\mathrm{x}}_{\text {base }}^{\text {tip }}}{\partial \overline{\mathrm{q}}}
\end{aligned}
$$

Equations (9) and (14) provide expressions for the kinetic and potential energy of the system. We start with the classic definition of the Lagrange equations of motion. 


$$
\frac{\partial}{\partial \mathrm{t}}\left(\frac{\partial \mathrm{T}}{\partial \dot{\overline{\mathrm{q}}}}\right)-\frac{\partial \mathrm{T}}{\partial \mathrm{q}}+\frac{\partial \mathrm{V}}{\partial \mathrm{q}}=\overline{\mathrm{Q}}
$$

The first term in Equation (16) can be expanded using the chain rule.

$$
\frac{\partial}{\partial \mathrm{t}}=\frac{\partial}{\partial \overline{\mathrm{q}}} \frac{\partial \overline{\mathrm{q}}}{\partial \mathrm{t}}+\frac{\partial}{\partial \dot{\overline{\mathrm{q}}}} \frac{\partial \dot{\overline{\mathrm{q}}}}{\partial \mathrm{t}}
$$

Substituting Equation (17) into (16),

$$
\frac{\partial}{\partial \dot{\bar{q}}}\left(\frac{\partial \mathrm{T}}{\partial \dot{\bar{q}}}\right) \ddot{\bar{q}}+\frac{\partial}{\partial \overline{\mathrm{q}}}\left(\frac{\partial \mathrm{T}}{\partial \dot{\bar{q}}}\right) \dot{\bar{q}}-\frac{\partial \mathrm{T}}{\partial \overline{\mathrm{q}}}+\frac{\partial \mathrm{V}}{\partial \overline{\mathrm{q}}}=\overline{\mathrm{Q}}
$$

As with the velocity computation in Equation (10), we can exploit the jacobian() function in Matlab ${ }^{\circledR}$ for the evaluation of many of the terms in Equation (18). First, the term $\partial \mathrm{T} / \partial \dot{\overline{\mathrm{q}}}$ is the differential of the scalar kinetic energy term with respect to the full state velocity vector defined in Equation (10). This results in the vector, $\mathrm{L}_{\mathrm{v}}$, in the script files in Appendix $\mathrm{A}$ and $\mathrm{B}$. The first term in Equation (18) represents the mass matrix. This expression is easily computed by taking the Jacobian on $\mathrm{L}_{\mathrm{V}}$ with respect to the full state velocity vector. The remaining elements in Equation (18) represent the nonlinear dynamics (coriolis, centripetal, gravitational) of the system. Thus, it should be clear that once the kinetic and potential energy terms are defined, it is straightforward to symbolically evaluate the dynamic equations of motion using Matlab's jacobian() function. The Jacobian for projecting external forces to the generalized coordinates can similarly be computed using the tip position of the robot and the jacobian function.

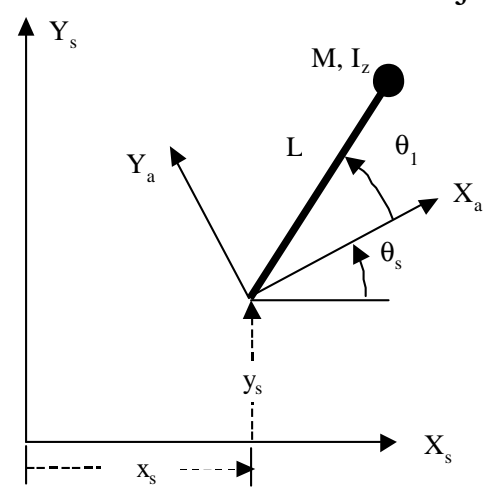

Figure 4: One DOF Model

We provide two examples to verify this methodology: a simple one degree of freedom system operating in a plane and a three degree of freedom system experiencing all six degrees of motion from the sea state. The first example is simple enough to verify through hand calculations. The second example is more complex, yet practical. Figure 4 shows the basic kinematic model of the one degree of freedom system experiencing 3 sea states in the X-Y plane. We are assuming a one DOF system with mass M and rotary inertia $I_{z}$ located at the tip of a link of length $\mathrm{L}$. The system is experiencing only three of the six sea states: surge $\left(\mathrm{x}_{\mathrm{s}}\right)$, heave $\left(\mathrm{y}_{\mathrm{s}}\right)$, and pitch $\left(\theta_{\mathrm{s}}\right)$. The only external force applied to the system is a joint torque, $\tau$, applied at joint 1. Appendix A shows the listing of code used to generate the dynamic equations of motion. The two output variables of interest are the MassMatrix and NLT. The resulting output is listed in Equation (19) and can be easily verified by the reader. Clearly, for this simple case, there is not a great advantage to using a symbolic package over hand calculations. 


$$
\begin{aligned}
& \text { MassMatrix }=\mathrm{M} * \mathrm{~L}^{2}+\mathrm{I}_{1 \mathrm{z}} \\
& \mathrm{NLT}=\mathrm{M} * \mathrm{~L}^{2} \ddot{\theta}_{\mathrm{p}}+\mathrm{I}_{1 \mathrm{z}} \ddot{\theta}_{\mathrm{p}}+\mathrm{M} * \mathrm{~L} 1 * \cos \left(\theta_{\mathrm{p}}+\theta_{1}\right) \ddot{\mathrm{y}}_{\mathrm{s}}-\mathrm{M} * \mathrm{~L} 1 * \sin \left(\theta_{\mathrm{p}}+\theta_{1}\right) \ddot{\mathrm{x}}_{\mathrm{s}}+\mathrm{M} * \mathrm{~g} * \mathrm{~L} 1 * \cos \left(\theta_{\mathrm{p}}+\theta_{1}\right) \\
& \text { combining } \\
& \left(\mathrm{M} * \mathrm{~L} 1^{2}+\mathrm{I}_{1 \mathrm{z}}\right)\left(\ddot{\theta}_{1}+\ddot{\theta}_{\mathrm{p}}\right)+\mathrm{M} * \mathrm{~L} 1 * \cos \left(\theta_{\mathrm{p}}+\theta_{1}\right) \ddot{\mathrm{y}}_{\mathrm{s}}-\mathrm{M} * \mathrm{~L} 1 * \sin \left(\theta_{\mathrm{p}}+\theta_{1}\right) \ddot{\mathrm{x}}_{\mathrm{s}}+\mathrm{M} * \mathrm{~g} * \mathrm{~L} 1 * \cos \left(\theta_{\mathrm{p}}+\theta_{1}\right)=\tau_{1}
\end{aligned}
$$

The power of this approach is more evident as we progress to more complex systems. Additional degrees of freedom only require additional homogeneous transforms. As a second example, we derive the dynamic equations of motion for the 3 degree of freedom system, shown in Figure 5, with the full six degrees of freedom from the sea state. A listing of the code used for computing the dynamics of the strength amplifying machine on the deck of a ship is shown in the listing in Appendix B. It should be clear comparing the listings in Appendix A and B that there is only a slight difference in the formulation of the transformations, but the methodology for deriving the dynamics is the same. The resulting equations of motion can be partitioned into a compact form, Equation (20),

$$
\left[\begin{array}{ll}
\mathrm{M}_{\mathrm{rr}} & \mathrm{M}_{\mathrm{rs}} \\
\mathrm{M}_{\mathrm{rs}}^{\mathrm{t}} & \mathrm{M}_{\mathrm{ss}}
\end{array}\right]\left\{\begin{array}{l}
\ddot{\overline{\mathrm{q}}}_{\mathrm{r}} \\
\ddot{\overline{\mathrm{q}}}_{\mathrm{s}}
\end{array}\right\}+\left\{\begin{array}{l}
\mathrm{NLT}_{\mathrm{r}} \\
\mathrm{NLT}_{\mathrm{s}}
\end{array}\right\}=\left\{\begin{array}{c}
\overline{\mathrm{Q}}_{\mathrm{r}} \\
\overline{0}
\end{array}\right\}+\left\{\begin{array}{c}
\mathrm{J}^{\mathrm{t}}\left(\mathrm{q}_{\mathrm{r}}\right) \mathrm{F}_{\mathrm{ext}} \\
\overline{0}
\end{array}\right\}
$$

where $M_{r r}$ is the 3x3 mass matrix for the robot with respect to the robot's state acceleration, $M_{\mathrm{ss}}$ is the $6 \times 6$ mass matrix of the robot with respect to the sea state acceleration, $\mathrm{M}_{\mathrm{rs}}$ is the inertial coupling of the sea state to the robot state, NLTr is a $3 \times 1$ vector of the nonlinear terms (gravitational, Coriolis, centripetal) as a function of both the robot's state and the sea state, $\mathrm{Q}_{\mathrm{r}}$ is the joint force input vector to the system, $\mathrm{F}_{\mathrm{ext}}$ is an external force vector applied to the end effector and $\mathrm{J}^{\mathrm{t}}\left(\mathrm{q}_{\mathrm{r}}\right)$ is the Jacobian from the end effector to the joint space. In order to include the dynamic equations of motion in Simulink ${ }^{\circledR}$, we use Equation (21) to solve for the acceleration of the robot's state vector as a function of all of the inputs (external forces and joint torques), system state (position and velocity) and external disturbances (sea state position, velocity, and acceleration).

$$
\ddot{\overline{\mathrm{q}}}_{\mathrm{r}}=\mathrm{M}_{\mathrm{rr}}^{-1}\left\{\overline{\mathrm{Q}}_{\mathrm{r}}+\mathrm{J}^{\mathrm{t}}\left(\mathrm{q}_{\mathrm{r}}\right) \mathrm{F}_{\mathrm{ext}}-\mathrm{NLT}_{\mathrm{r}}-\mathrm{M}_{\mathrm{rs}} \ddot{\overline{\mathrm{q}}}_{\mathrm{s}}\right\}
$$

While the output of the single degree of freedom, planar case can be listed in Equation (19), the results of the dynamic equations of motion for the second system generates 84 pages of c-code and would require considerable effort to derive by hand. One primary concern is the validity of the results. For now, we can only verify the basic methodology by comparing to simple cases. To date, we have compared the methodology to a number of manipulators with stationary bases and achieve the same symbolic results. In addition, we have considered only simple one and two degree of freedom systems experiencing one to three degrees of ship motion. In each case, the symbolic solutions are the same leading us to believe the methodology is sound. An obvious question is "what can you do with 84 pages of c-code?" Fortunately, the code can be directly imported into Simulink ${ }^{\circledR}$ through the S-Function builder. Finally, one might ask "how long does it take to simulate a system with 84 pages of c-code?" The simulation was surprisingly fast. It takes 178 seconds to execute a 120 second simulation with a fixed 0.01 second time step and $4^{\text {th }}$ order Runge-Kutta integration. This was executed on a $750 \mathrm{MHz}$ Pentium III laptop. The motivation for computing the dynamics equations of motion are two fold. First, by having the dynamics in a symbolic form, it is possible to aid in the design process, changing parameters to optimize the system. Second, a model of the system dynamics can aid in increasing the fidelity of simulation for control design and analysis. 


\section{Simulation Platform}

The objective of this research is to develop advanced control methodologies to reduce the influence ship motion disturbances have on position and force controlled manipulation systems. As a preliminary approach, we use detailed simulation models to explore the impact wave motion has on the tracking performance of general manipulation systems. To increase the fidelity of the simulation, we include not only the mechanical dynamics, detailed in the previous section, but include the nonlinear dynamics associated with the actuation. Since our motivation is to develop advanced control methodologies for strength amplification systems, our target systems have similar components that are generally found on Human Amplification Technology (HAT). The system modeled in this investigation is displayed in Figure 5. This system has a 500-pound payload capacity and has three active degrees of freedom. The actuator models include nonlinear dynamic modeling of the hydraulic system (servo-valve orifice equations equations, asymetric cylinders, fluid compliance ...), controls and the dynamic equations of motion computed above.

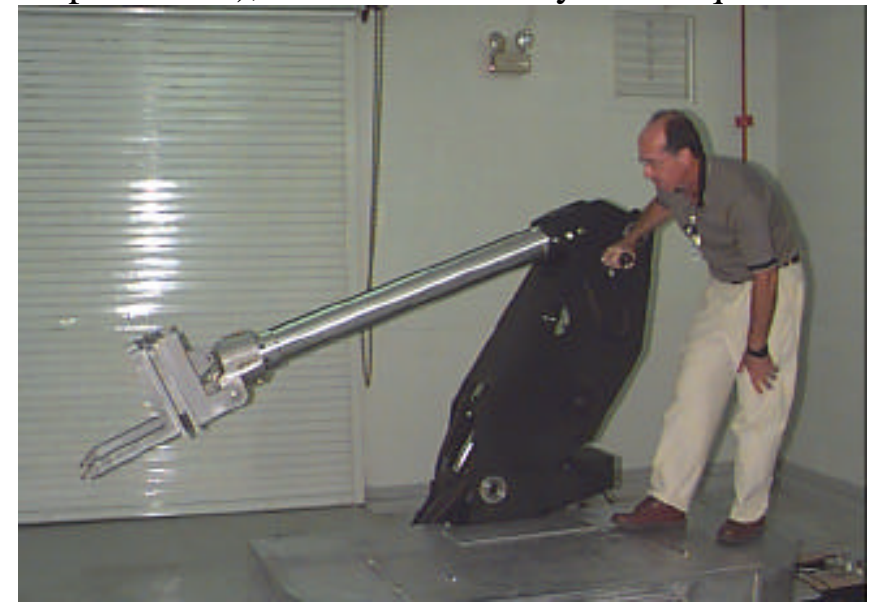

Figure 5: Strength Amplifying Machine

The hydraulic actuator models generate force as a function of the servovalve current, actuator position and velocity. The Simulink ${ }^{\circledR}$ model of the full HAT controlled system, including the sea state inputs, hydraulic models, controls, and dynamic equations of motion is shown in Figure 6. 


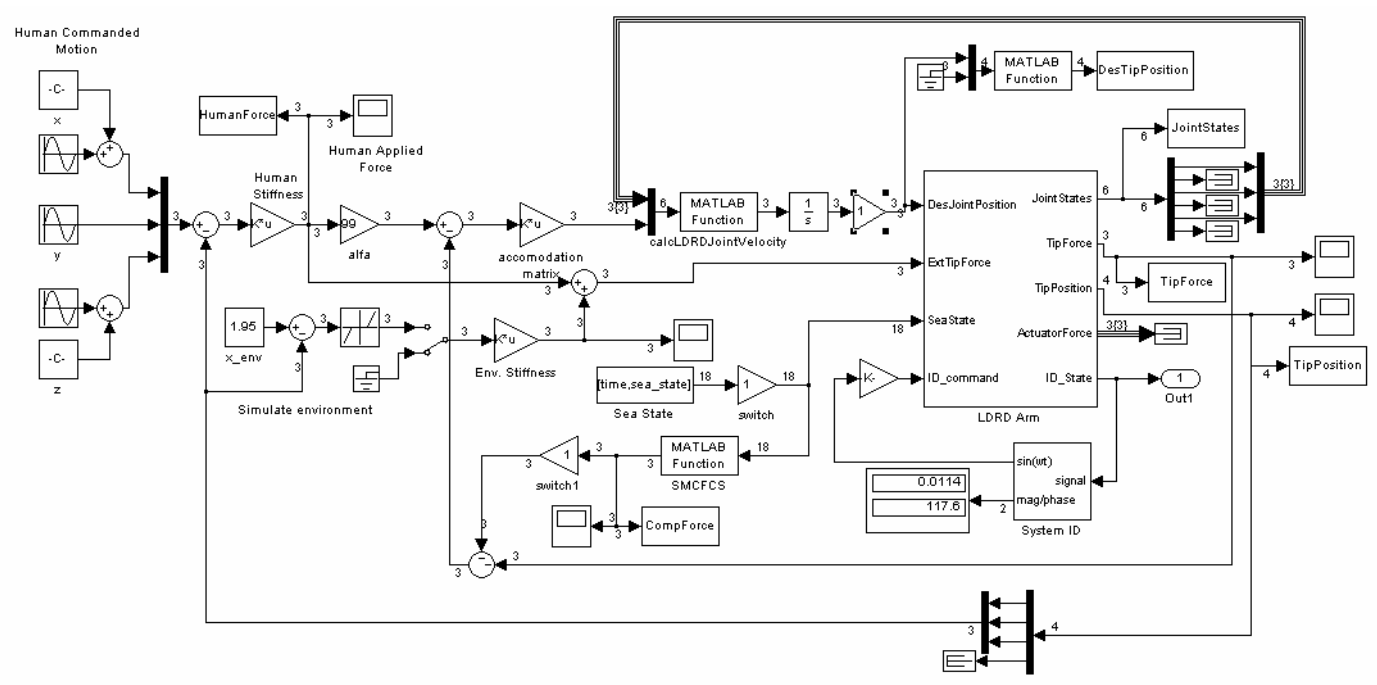

Figure 6: HAT Simulation Model

The nonlinear model of the hydraulic manipulator, shown as the LDRD Arm block in Figure 6, is expanded in Figure 7. This model includes auxiliary inputs for system identification. However, the inputs to the model include the desired joint positions, the eighteen elements of the sea state (displacement, velocity and acceleration of roll, pitch, yaw, heave, surge, and sway), and the external force applied to the robot (from human and/or environment).

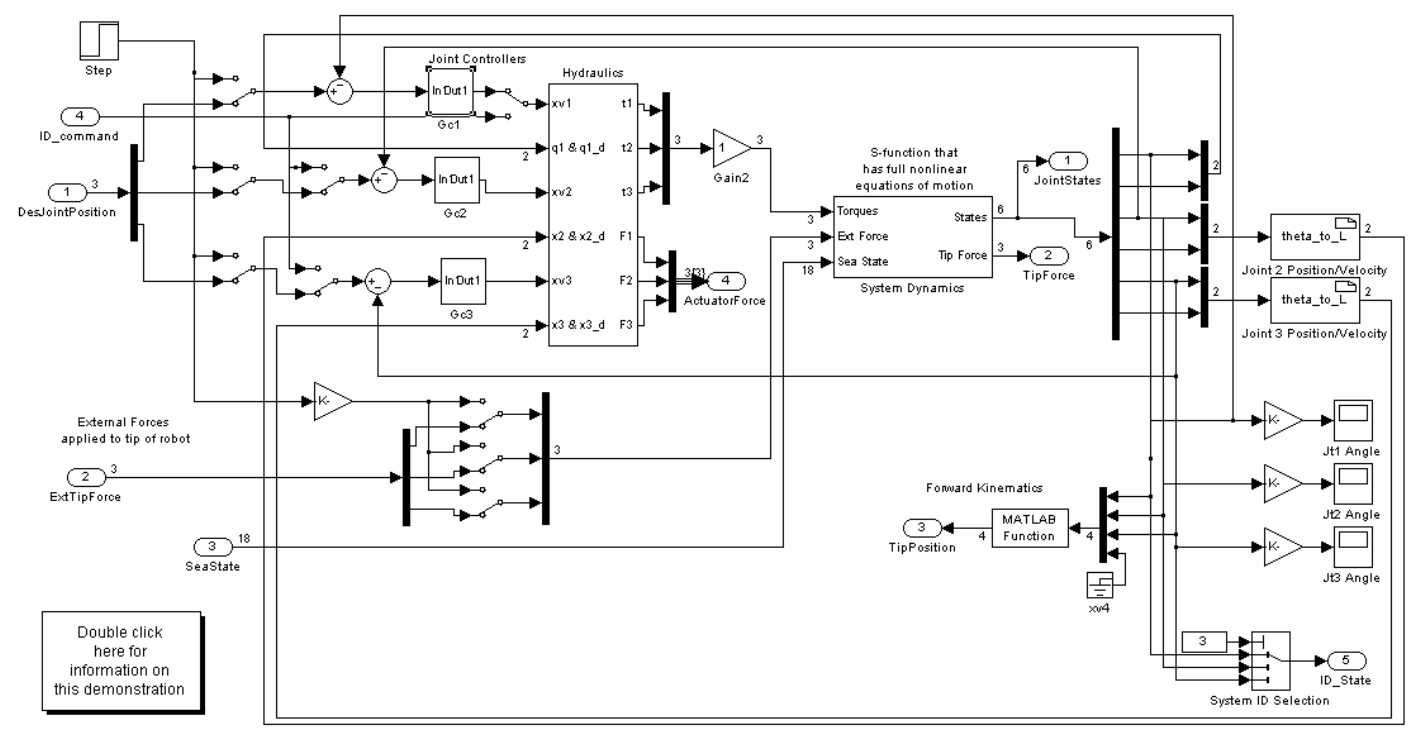

Figure 7: Details of HAT Controller and Manipulator Model

There are two primary blocks to note in Figure 7. The first is the System Dynamics block. This contains the c-code generated previously that represents the forward dynamics of the LDRD arm. The second is the Hydraulics block, expanded in Figure 8. From the expanded hydraulics block, each of the three joints has two primary elements: the servo valve and the actuator. In the case of the second and third joint, there is also a transmission associated with the coupling of the linear actuator with the joint. The 
servo valve models, shown in Figure 9, are based on the full nonlinear orifice equations and regulate the fluid flow to the actuators as a function of excitation signal (command to the moving coil on the servo vavle), the supply and return pressure, as well as the pressure on both sides of the actuator. The actuator model, Figure 10, generates a force based on the position, velocity, and bulk modulus (stiffness) of the fluid. The position and velocity of the actuator come from the dynamic model of the manipulator. The force from the actuator is the excitation to the dynamic model of the manipulator. There are three elements to the joint controllers, one of which is shown in Figure 11. There is the general joint compensator, the repetitive learning controller (which can be switched on or off), and an inner pressure control loop (shown in the actuator models). Hydraulic actuators are generally characterized as type one systems with a lightly damped pair of poles. The pressure control feedback controls the joint damping, much like velocity feedback on an electric motor. The joint position compensators provide adequate motion tracking, with a target closed loop bandwidth approaching $3 \mathrm{~Hz}$, the nominal bandwidth of gross human motion. Finally, the repetitive learning controller, detailed shortly, allows easy integration of the learning control with the joint compensator. It should now be clear that, to the best of our ability, the full nonlinear behavior of the manipulator is embodied in the simulation of the manipulator. We now will explore the impact the sea state has on the position and force tracking capability of the manipulator. 

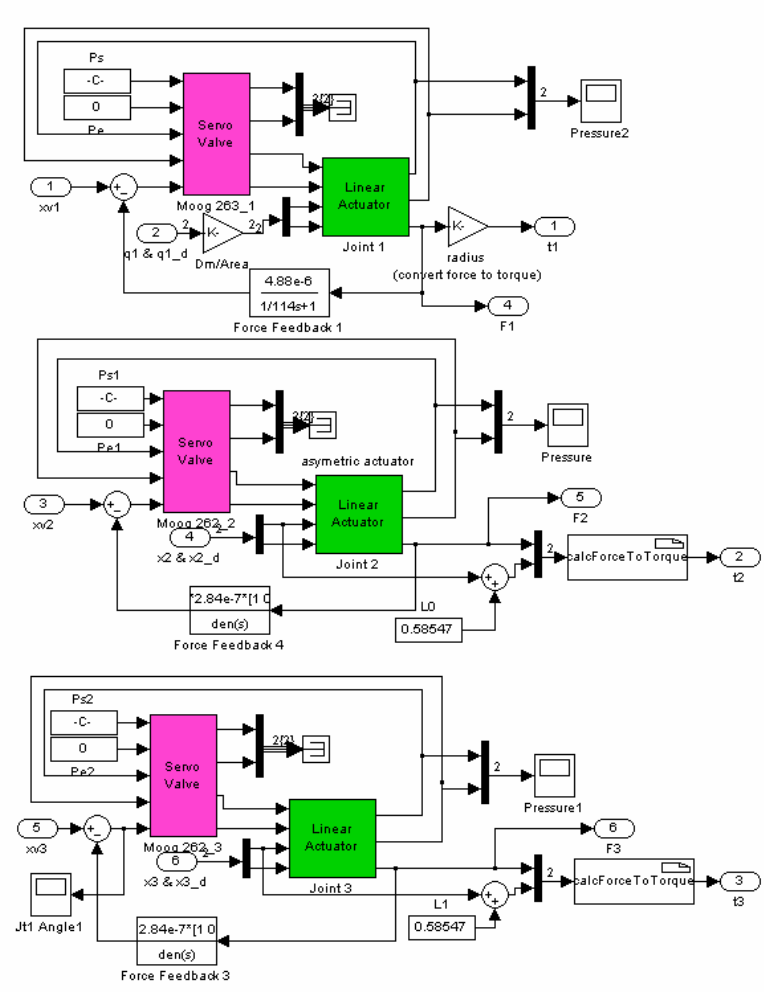

Figure 8: Details of Hydraulic Actuators

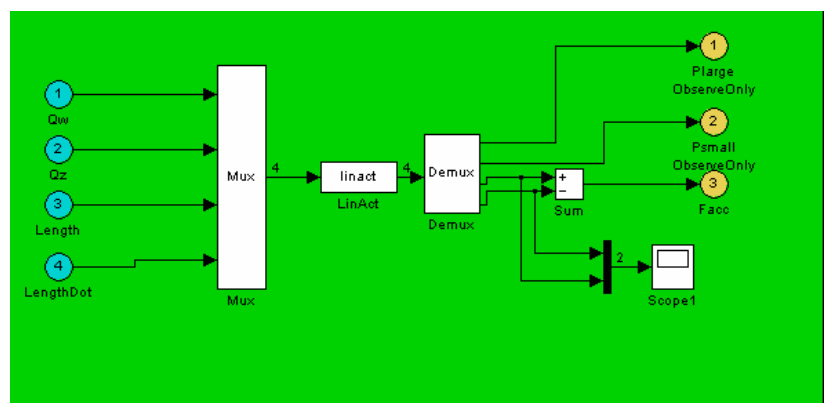

Figure 10: Linear Actuator Model

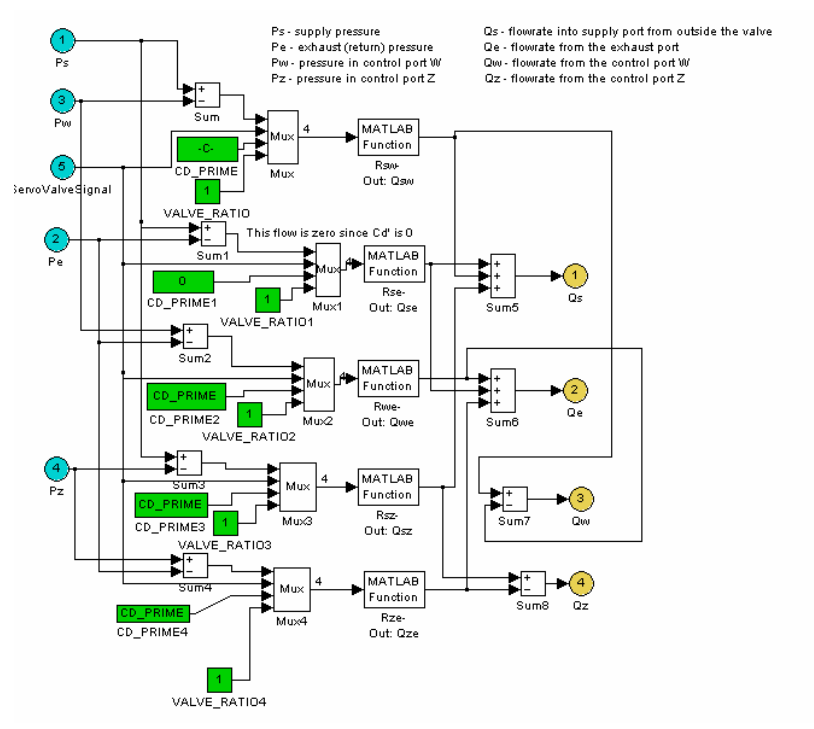

Figure 9: Details of Servovalve

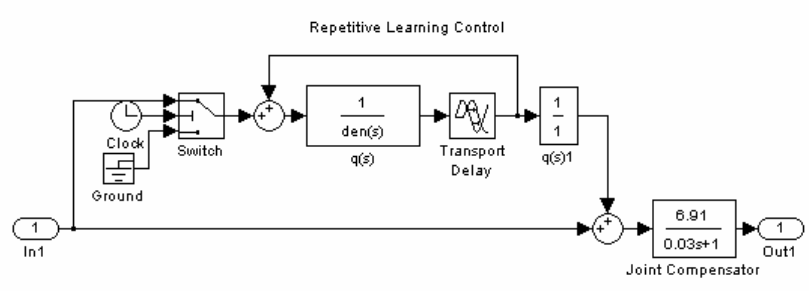

Figure 11: Joint Compensator

\subsection{Impact of Sea State on Conventional Control}

We now consider the impact sea state has on the performance of a HAT system. By performance, we mean accuracy of tip position and perturbations on the force experienced by the operator. Inputs to the system consist of a human command regulating about a single point and a sea state with conditions commensurate to a destroyer moving at 20 knots in sea state 5. Figure 12 shows the force provided by the human while attempting to regulate the tip position. There is the expected DC bias on the Z-direction force required to offset the gravitational load. The payload weight is $2224 \mathrm{~N}$ which projects to a human force of 22.4 $\mathrm{N}$ when in static equilibrium (as illustrated in Figure 35). However, the maximum perturbation, after the initial transient, during a 120 second simulation run was $11.38 \mathrm{~N}$, approximately $51 \%$ of the actual load. 
In addition, there are orthogonal disturbances in the $\mathrm{X}$ and $\mathrm{Y}$-directions due to the rolling, swaying and surging motion of the ship. The resulting tip motion is displayed in Figure 14, Figure 15, and Figure 16. It is clear that the vertical direction is the most sensitive to the sea state. The variation in the vertical tip position is $22.8 \mathrm{~mm}$. The tracking error above is a function of two inputs: the force (due to the sea state) applied to the arm and the commanded motion from the force applied by the operator. In the following sections, we will explore alternative control strategies that address compensation of these disturbance forces in order to increase the positioning accuracy and reduce the disturbance force reflected to the operator.

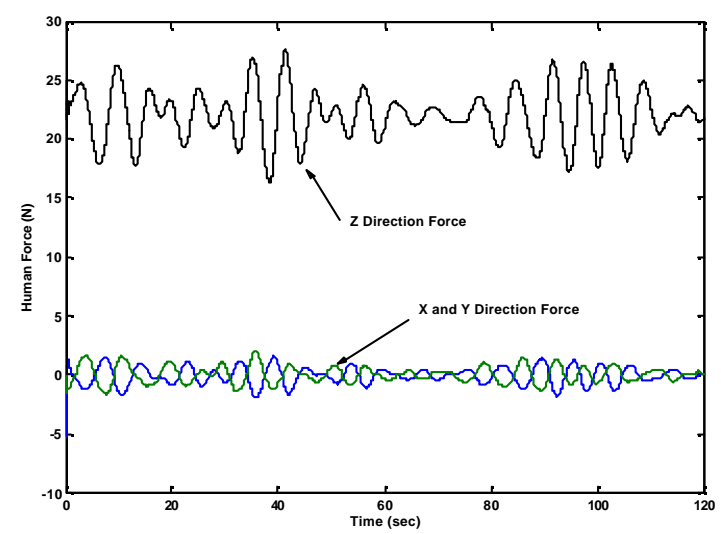

Figure 12: Human Force w/out Compensation

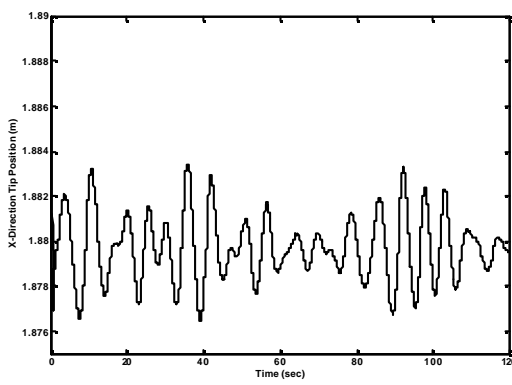

Figure 14: X Tip Direction

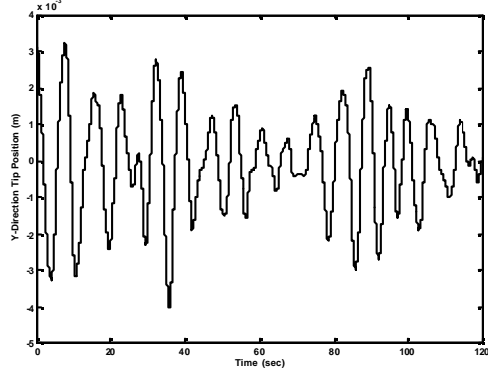

Figure 15: Y Tip Direction

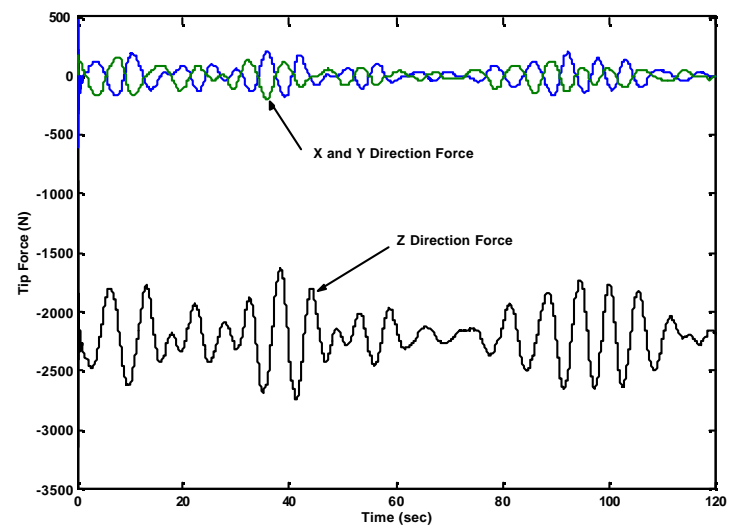

Figure 13: Tip Force w/out Compensation

\subsection{Preliminary Experiments with Repetitive Learning Control}

This investigation explores control strategies for both position and force controlled systems experiencing severe sea state disturbances. In the previous section, we illustrated the impact of sea state disturbances on a force controlled system, emphasizing the disturbance reflected to the operator and reduction in positioning resolution. Emphasis now shifts to alternative control strategies for attenuating the sea state disturbance on position and force controlled systems. The first series of experiments concentrates on position controlled systems. For our first simulation, we start with a sinusoidal model with a fixed frequency for each of the six sea states. We assume a significant wave height of $7 \mathrm{ft}$ and average period of 6 seconds (sea state 5). For demonstation purposes, the system has a $500 \mathrm{lb}$ payload, has linear position control with a gain margin of $10 \mathrm{~dB}$ and phase margin of 60 degrees, and is commanded to regulate the initial joint configuration. Under these sea states, the vertical and horizontal tracking error (displayed in Figure 18, Figure 20, and Figure 22) exceed 1 inch. We then introduce a Repetitive Learning Controller (RLC) with a fixed delay $\left(\mathrm{T}_{\mathrm{d}}\right)$ that is the same as our simulated wave period. Details on the design of an 
RLC, see Figure 17, (specifically the filters $q(\mathrm{~s})$ and $\mathrm{b}(\mathrm{s})$ ) can be found elsewhere in the literature.[17,18] Without the RLC (Figure 18, Figure 20, and Figure 22), the amplitude of the tip position error is $1.3 \mathrm{~mm}$ in the $\mathrm{X}$ direction, $3.05 \mathrm{~mm}$ in the Y-direction and $4.05 \mathrm{~mm}$ in the Z-direction. With the RLC (see Figure 19, Figure 21, and Figure 23), the error reduces to $0.11 \mathrm{~mm}$ in the $\mathrm{X}$-direction, $0.23 \mathrm{~mm}$ in the $\mathrm{Y}$-direction and $0.33 \mathrm{~mm}$ in the Z-direction. These results would indicate a clear advantage to using RLC for compensating for the periodic disturbance of wave motions. However, while the sea state, as illustrated in Figure 1, is generally characterized as periodic and sinusoidal, there is some noticable varition in the amplitude and frequency of the ship motion.

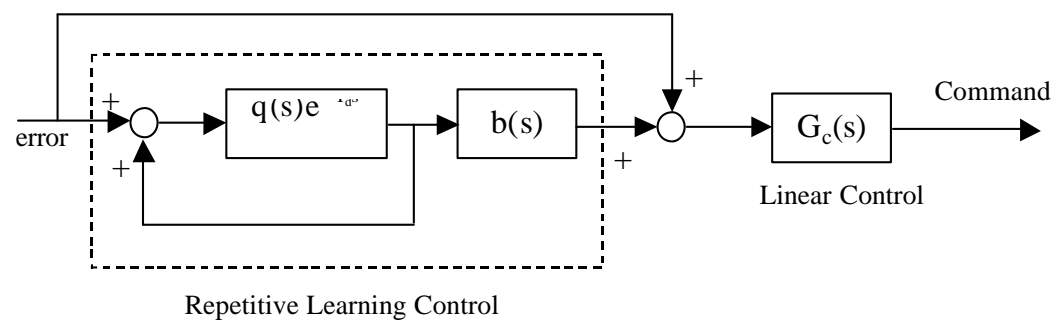

Figure 17: RLC Joint Control Strategy

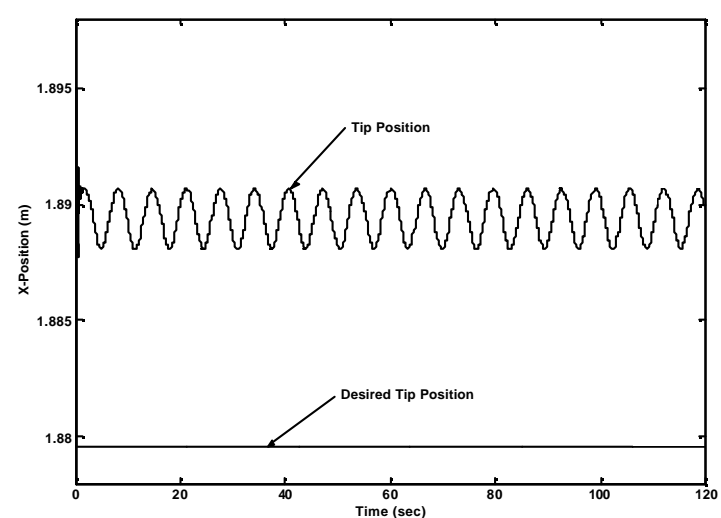

Figure 18: X-Position, simple waves, no compensation

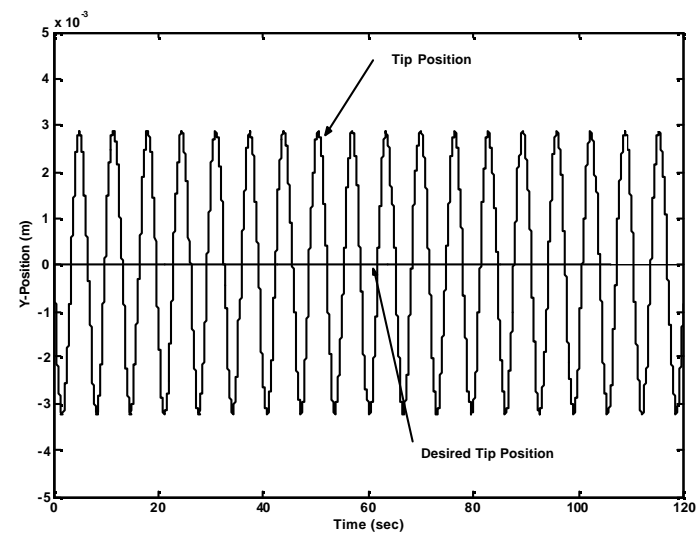

Figure 20: Y-Position, simple waves, no compensation

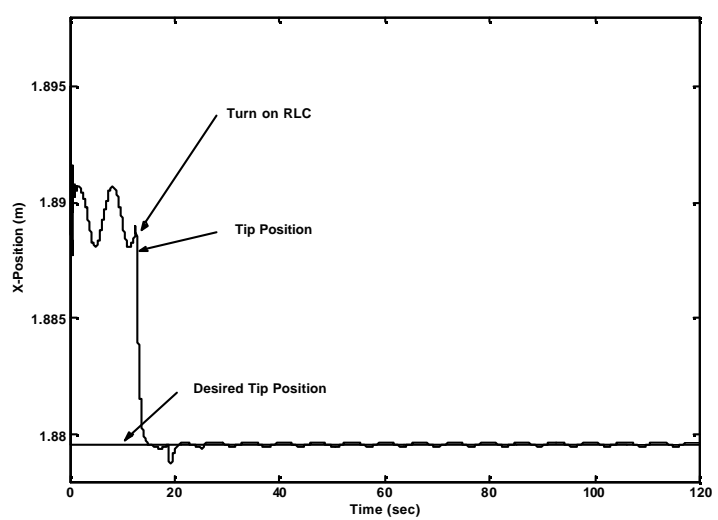

Figure 19: X-Position, simple waves, with RLC

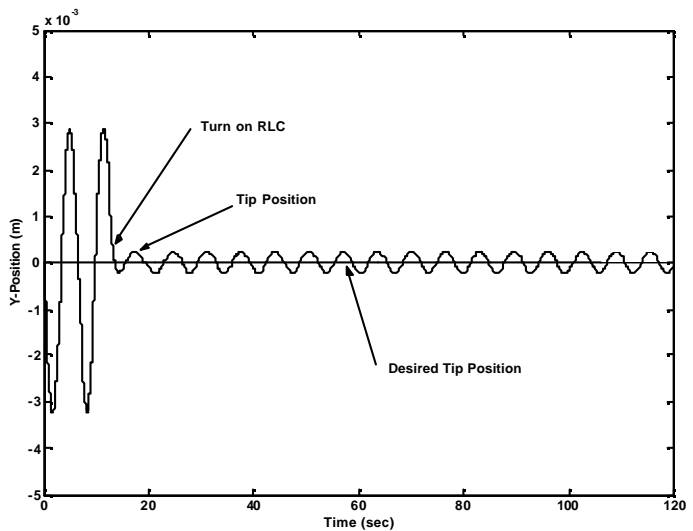

Figure 21: Y-Position, simple waves, with RLC 


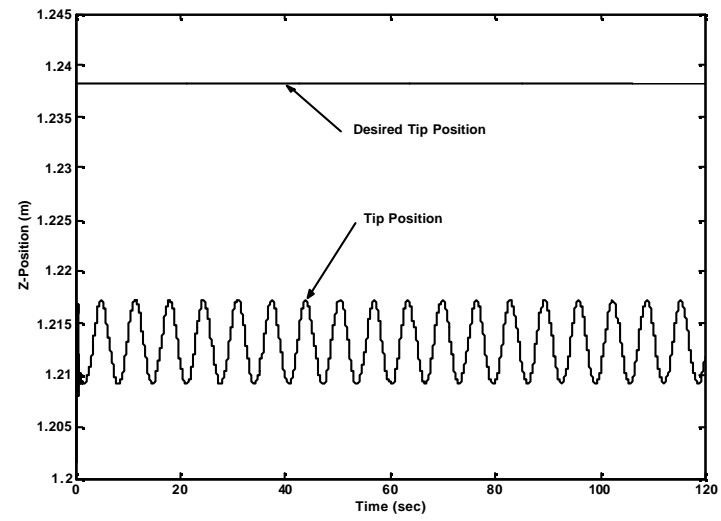

Figure 22: Z-Position, simple waves, no compensation

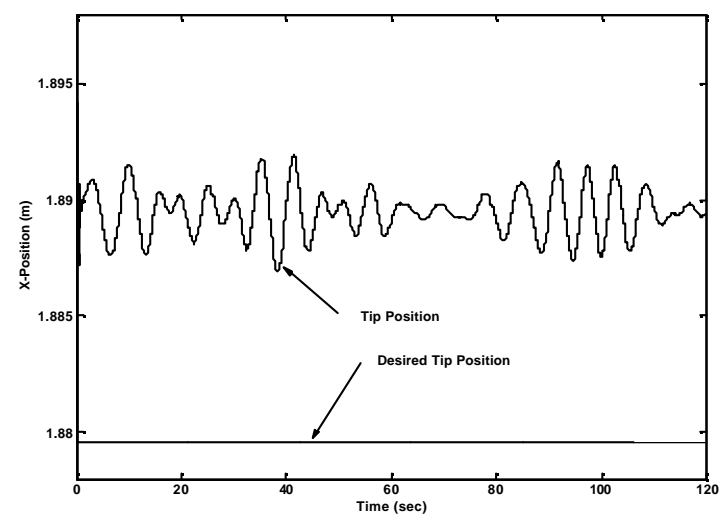

Figure 24: $\mathrm{X}$-Position with realistic wave motion

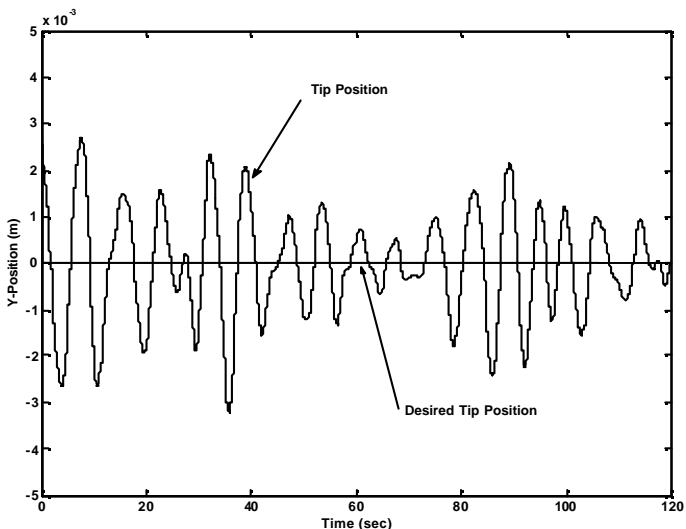

Figure 26: Y-Position with realistic wave motion

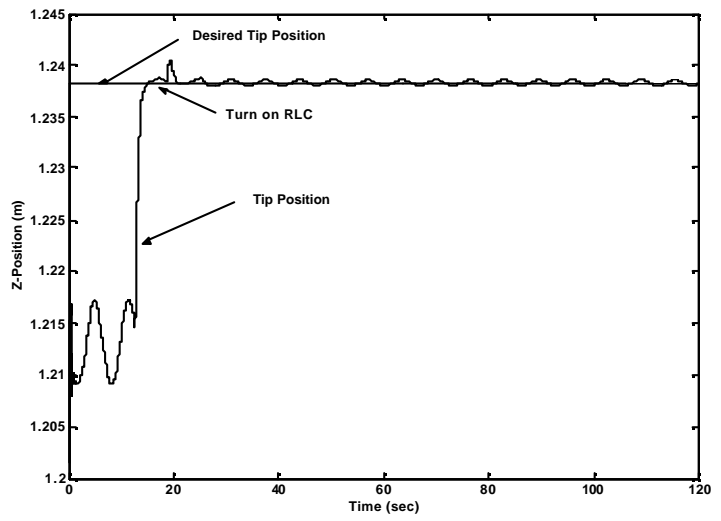

Figure 23: Z-Position, simple waves, with RLC

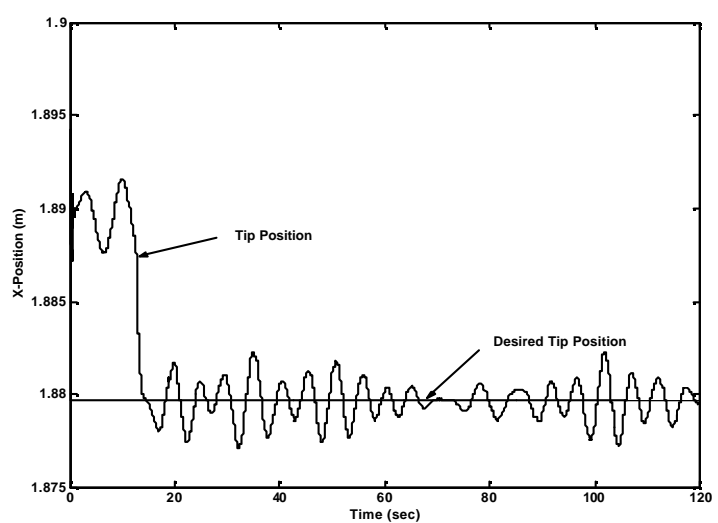

Figure 25: X-Position with RLC and realistic wave motion

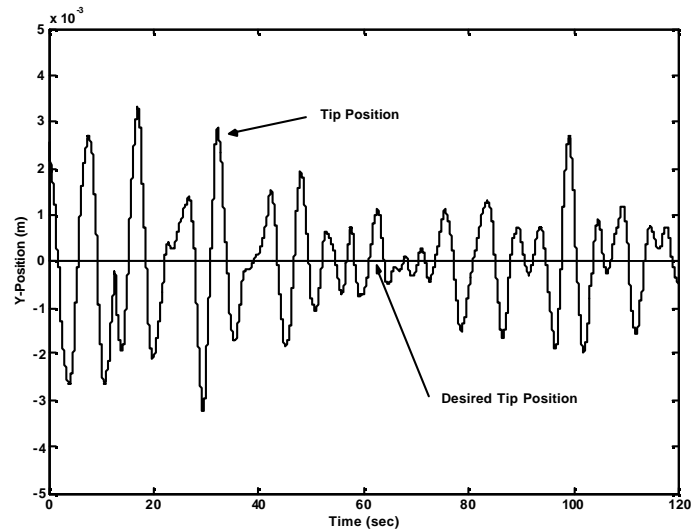

Figure 27: Y-Position with RLC and realistic wave motion 


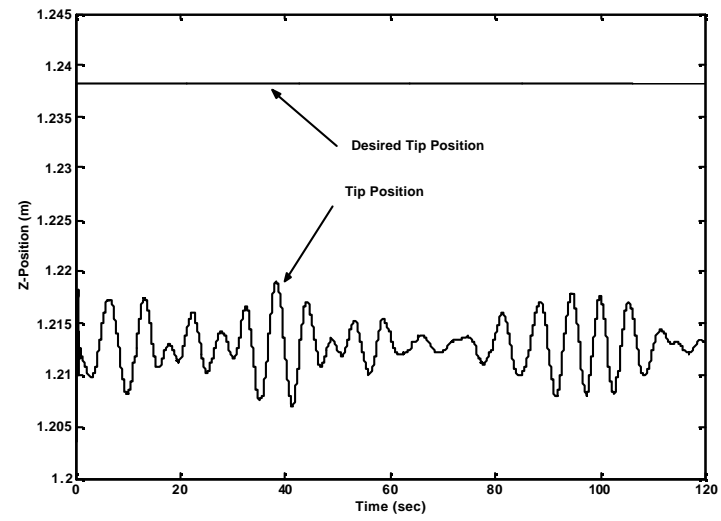

Figure 28: Z-Position with realistic wave motion

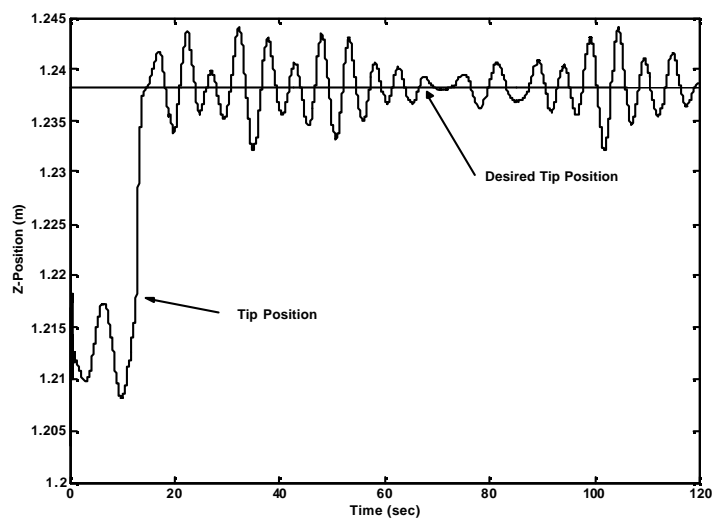

Figure 29: Z-Position with RLC and realistic wave motion

We now repeat the same series of simulations. However, we replace the periodic wave disturbance with a more representative disturbance generated by the SMP program. Figures 24, 26 and 28 show the response of the system using the same conventional control as used previously. Likewise, Figures 25,27 , and 29 show the system response when using a fixed period RLC algorithm. Clearly, the variations in the wave period negatively impact the performance of the fixed time delay RLC. There are some time segments (from 90 to 120 seconds) where it appears there is some benefit to using RLC. However, the variable nature of the wave period degenerates the tracking capacity of the RLC. Attempts were made to track and adapt to the variable frequency of the disturbance. However, the disturbance frequency does not vary slowly with respect to time and making adaptation futile. A detailed analysis of the sensitivity of RLC is provided in Appendix C.

\section{MARitime Force CONTROL}

Our emphasis now shifts towards force control with specific emphasis on the impact of sea state disturbances on human amplification systems. We begin with a basic description of human strength amplification technology and include simulation results to demonstrate the basic characteristics of a general strength amplification system, specifically human strength amplification and contact stability. This is followed by an investigation of the impact of sea state disturbances on HAT systems and an exploration of alternative control methodologies to mask these disturbances from the operator.

\subsection{Baseline Performance of HAT System Simulation}

We now transition from position controlled manipulation systems to force controlled systems. Our focus is on a Force-Force control strategy to realize strength amplification. We begin with a basic description of the HAT control strategy. Next, we show through simulation the characteristics of a HAT control architecture on a dynamic model of the system in Figure 5. Finally, we illustrate our primary problem: the influence sea states have on the performance of a HAT controlled system.

HAT controlled systems basically consist of a robot with two force inputs: the force from the payload and the force from the human. The force-force control strategy, Equation (22), amplifies the human force vector, $F_{h}(\alpha$ is the amplification factor), and strives to balance this amplified human force with the payload 
force, $\mathrm{F}_{\mathrm{e}}$.

$$
\Delta \mathrm{F}=\alpha \mathrm{F}_{\mathrm{h}}-\mathrm{F}_{\mathrm{e}}
$$

There are a wide variety of control strategies that enable force-force control and human amplification.[19] A few examples are illustrated in Figure 30, Figure 31 and Figure 32. The primary motivation is to control the manipulator so that it drives the force error to zero. However, there are many other aspects to the control of a strength amplification system beyond the tracking and amplificication of human forces. First, safety is always a primary concern. As an example, the Next Generation Munitions Handler, Figure 33, was designed to enable a single human to carry and manipulate a payload in excess of $2000 \mathrm{lbs}$. Contact stability and fault tolerence are high priorities. The control architecture should enable loss of human excitation (accidentally let go of system). Such systems are designed to provide human interaction with the payload and environment. Subsequently, the system requires a closed loop bandwidth similar to gross human movements (approximately $2 \mathrm{~Hz}$ ) as well as sub-millimeter positioning resolution. All of these characteristics must be considered in the control design. For this study, we adopt the Accomodation Control architecture. 


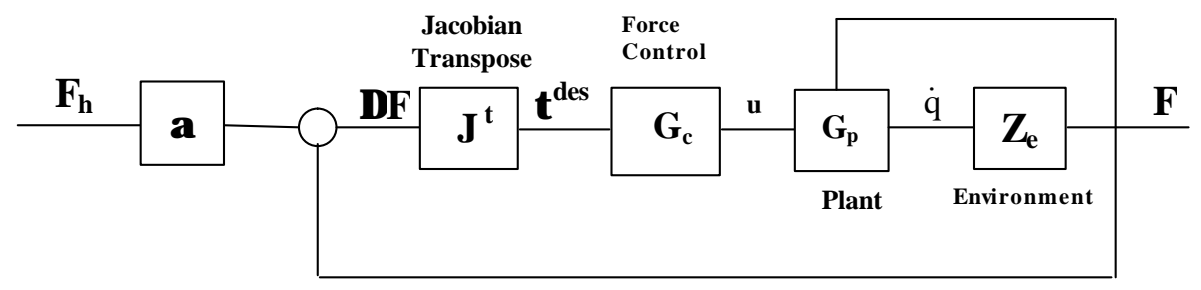

Figure 30: Explicit Force Control

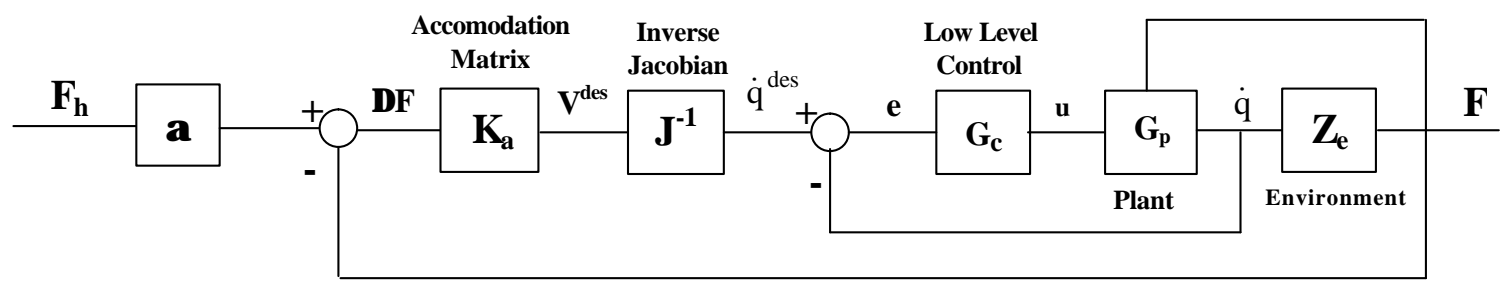

Figure 31: Accomodation Control

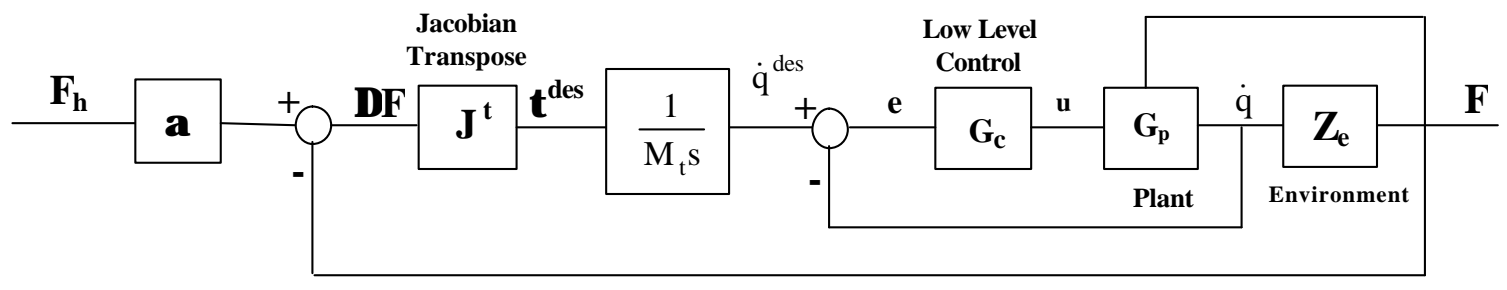

Figure 32: Acceleration Control

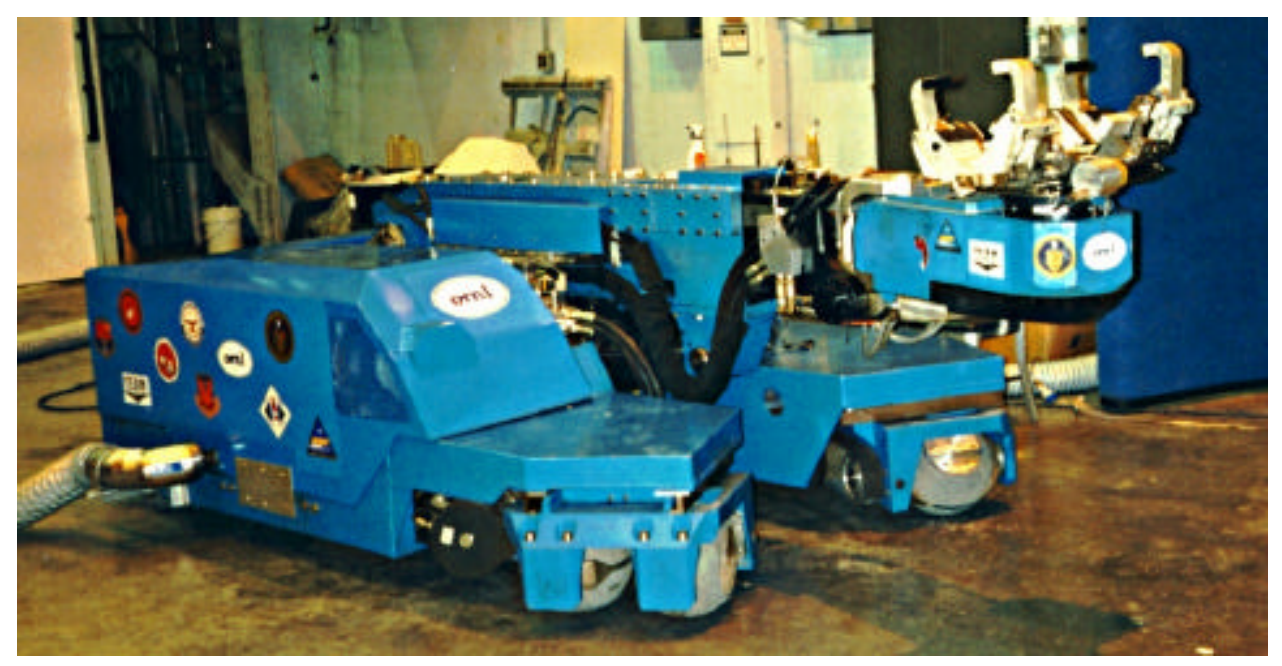

Figure 33: ORNL's Next Generation Munitions Handler

One of the challenges faced with this study is assessing the performance of a human controlled system through simulation. Human behavior and physical response is a complex phenomenon. However, Flash and Hogan suggested that planning multi-joint movements takes place in the Cartesian space rather than the joint 
space of the limbs. This approach, the equilibrium trajectory hypothesis, models human forces as a stiffness relationship between a target trajectory and actual trajectory of the limb[20]. Clearly, human stiffness varies from subject to subject, limb to limb, configuration to configuration. However, from the studies of Flash, a good approximation would be a diagonal stiffness matrix with a magnitude of $500 \mathrm{~N} / \mathrm{m}$. For our HAT controlled simulations, human commands are modeled, shown in the left portion of Figure 6 as a force vector that is the product of a human stiffness matrix and the cartesian tracking error (error between where the human wants the tip of the robot to be and the actual tip position of the robot). For the HAT control, the human force vector is amplified by the gain alpha and compared to the measured tip force of the robot. This force error is passed through an accomodation matrix, generating a desired tip velocity of the robot. The manipulator Jacobian provides the transformation from a desired tip velocity to the desired joint velocity, which is subsequently integrated and fed into the manipulators joint controller. This is the essense of the HAT control methodology. We will now provide further details into the modeling of the hydraulic actuators with the combination of the dynamic equations of motion described previously.

As a baseline, we consider the performance of the HAT system using only conventional control techniques. The first simulation simply looks at the force amplification, without any sea state disturbances. Figure 34 and Figure 35 display the tip and human applied force when the system starts. The initial transient is due to the immediate gravitational loading on the arm and quickly damp out. The amplified human force balances the tip force (due to the gravitational load of the payload) with the appropriate amplification factor of 100 .

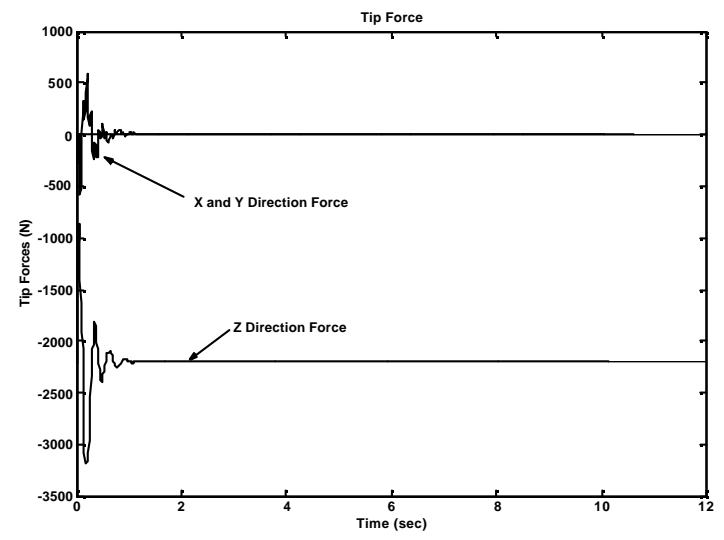

Figure 34: Tip force when stationary

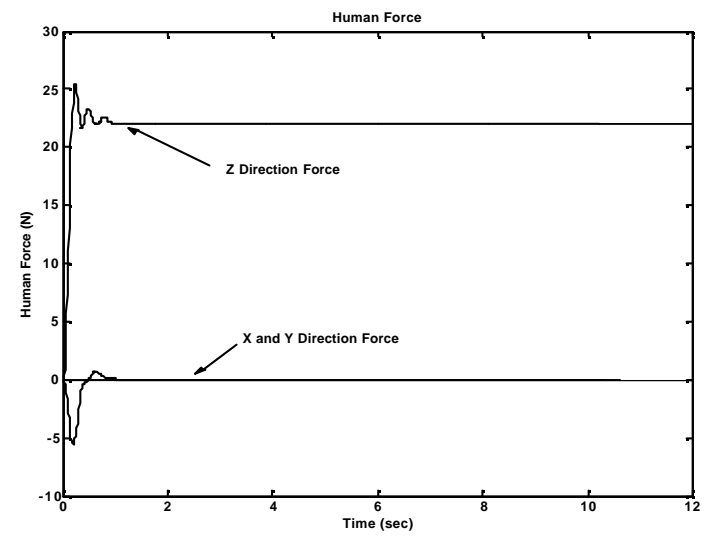

Figure 35: Human force when stationary

The next aspect of the HAT control is motion tracking. When unconstrained, the human applied force directs the motion of the arm, in Cartesian space, scaled through the accommodation matrix. In the simulation, we are interested in the decoupling of Cartesian motion. The motion of the tip should be in the direction of the force applied to the arm by the human. Figure 36 through Figure 41 show the response of the arm when isolating one Cartesian motion at the operator input. It is clear that, when unconstrained, the HAT system operates with the appropriate behavior. 


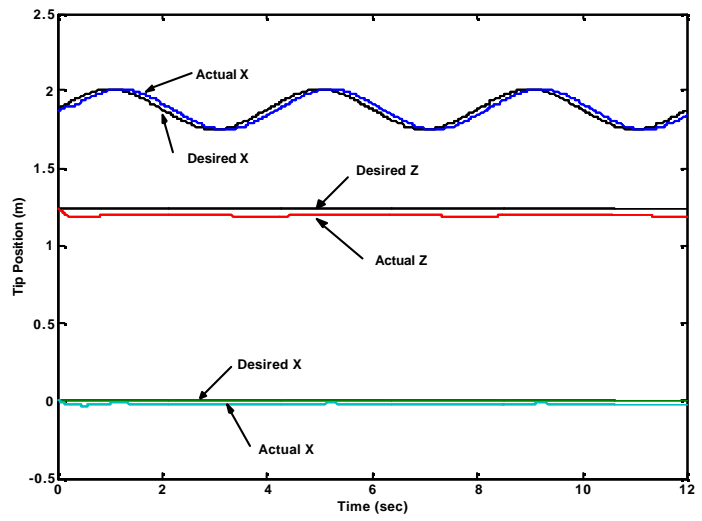

Figure 36: Tip Response to X-Direction Command

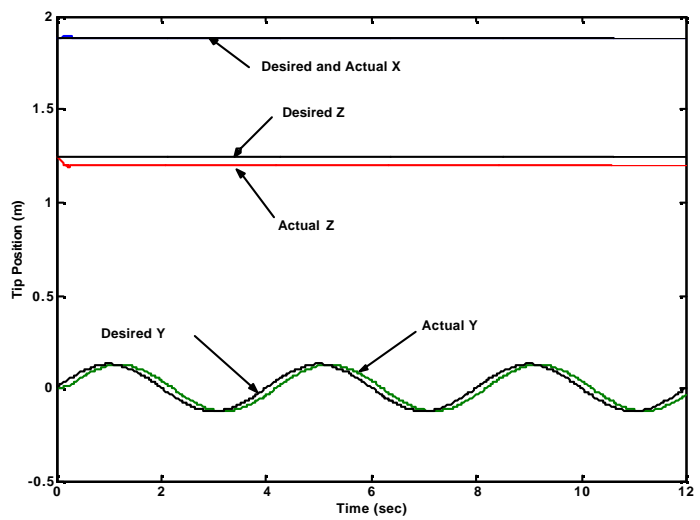

Figure 38: Tip Response to Y-Direction Command

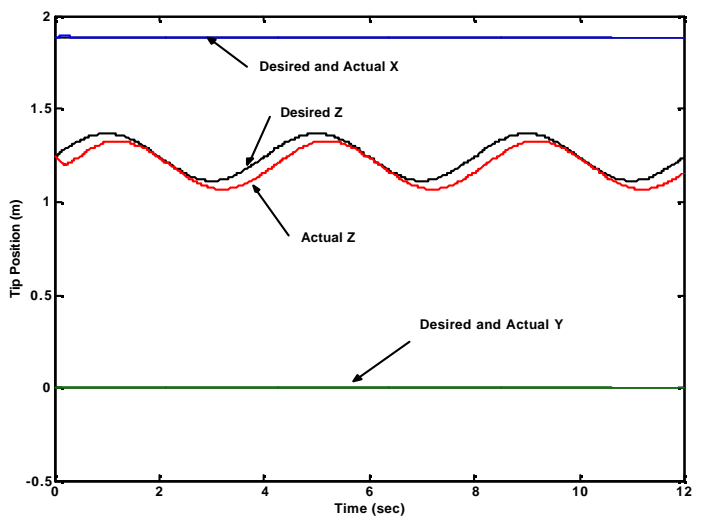

Figure 40: Tip Response to Z-Direction Command

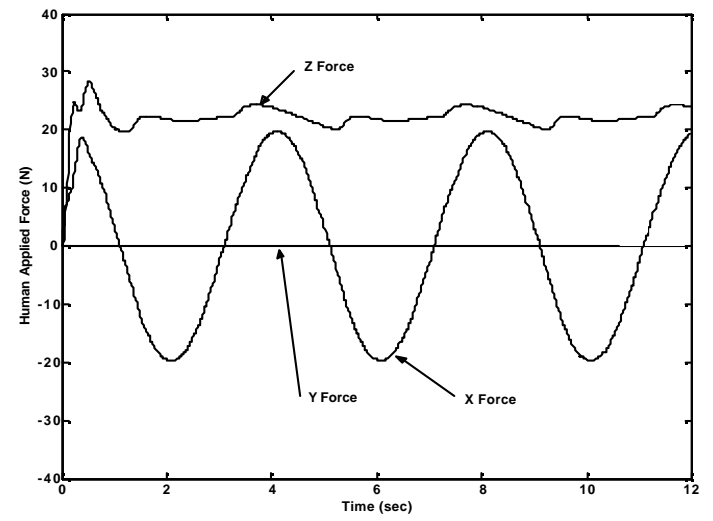

Figure 37: X-Direction Human Command

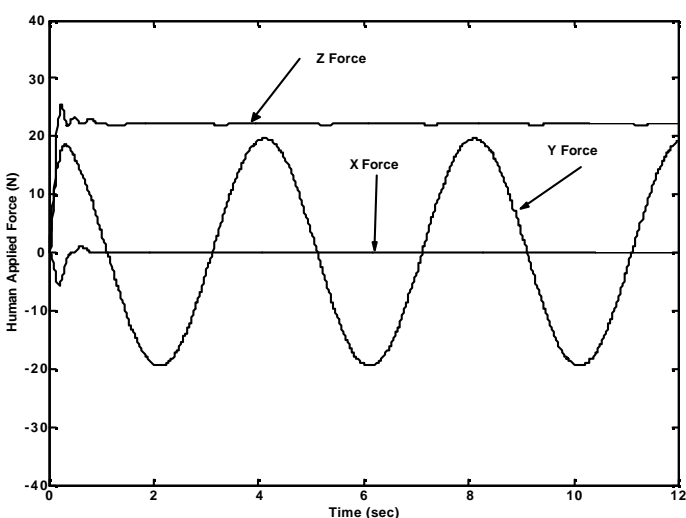

Figure 39: Y-Direction Human Command

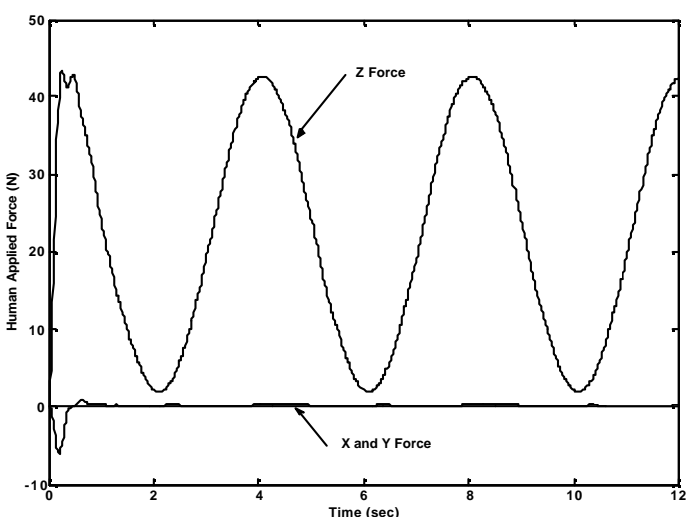

Figure 41: Z-Direction Human Command

One final concern is contact stability of the HAT system. When the system contacts a hard surface, it should not only be stable, but not exhibit oscillatory behavior. Any contact vibration should die out rapidly and not exhibit a bouncing characteristic. To test contact stability, we model the environment as a nonlinear spring representing a surface that has zero stiffness in one direction and large stiffness in the opposite direction. The operator commands motion in the y-direction. The trajectory consists of a trapezoidal trajectory in which the target position is slightly behind the wall, ensuring contact. The magnitude of the surface stiffness is set at the $1.12 \mathrm{e} 6 \mathrm{~N} / \mathrm{m}$ which is slightly larger than the mechanical stiffness of the last link 
of the arm. This should provide a good approximation for contact with a hard surface. Figure 42 shows the various stages of the human applied force. Figure 43 shows the corresponding tip position. There is an initial transient when the arm is loaded and all initial conditions are zero. Next, the operator commands a constant velocity in the y-direction. This command manifests itself as the momentum force profile from 3 to 4 seconds. The desired velocity is $65 \mathrm{~mm} / \mathrm{sec}$ during contact with the wall. The force continues to increase while the trajectory (representing the desired motion of the human) continues into the wall, and levels out. As desired, the human contact pressure (approximately $26 \mathrm{~N}$ ) is 100 times lower than the force measured in the $y$-direction on the manipulator. So, this series of simulations demonstrates contact stability with force amplification.

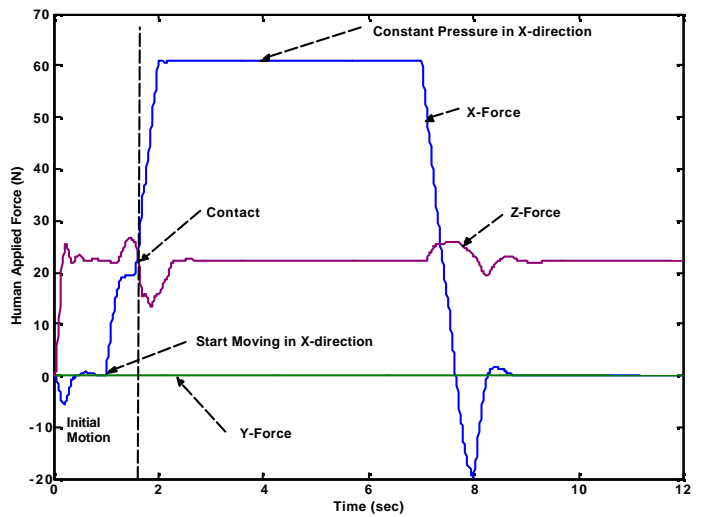

Figure 42: Human Force during contact

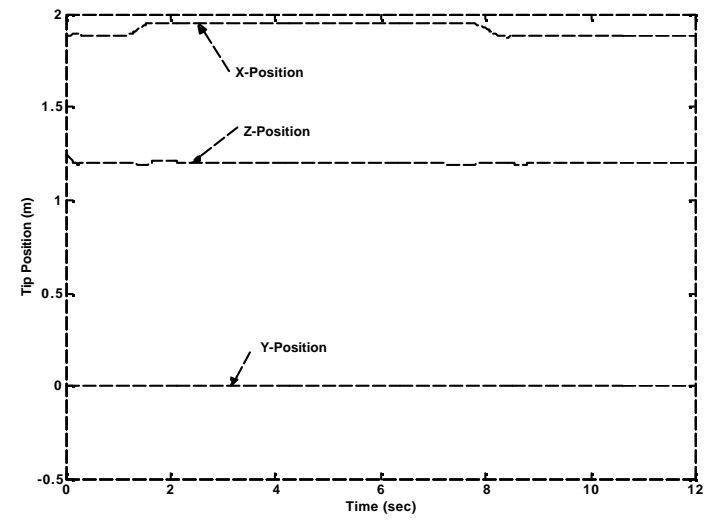

Figure 43: Tip position during contact task

\subsection{Ship Motion Compensation for Force Control Strategy}

In earlier sections, we illustrated the impact ship motion has on the tracking performance of a position controlled manipulation system. First, it was clear that the disturbance was low frequency in nature. Second, while the disturbance appears to be sinusoidal, the frequency is time varying. Our analysis and simulations illustrated the potential for Repetitive Learning Control if the period of oscillation was fixed. If the period of the disturbance was slowly varying, there is potential for adapting the target period. However, there may be significant variation in the period in less than 2 cycles of the disturbance complicating any attempt at RLC adaptation. The impact of ship motion becomes more significant when dealing with force control architectures. With position control, the disturbance forces due to ship motion only influences the system response through the dynamics of the manipulator. With a force control architectures, the force disturbance has potentially two avenues to influence system response: the system dynamics and the feedback of the disturbance force to the force control. Finally, with a HAT controlled system, there is the possibility for a third avenue for the disturbance force to influence the response of the system: the human command. Inertial (due to accelerations due to the sea state) and variations in the gravitational force (due to rolling and pitching motion of the ship) generate significant variations in the interaction force between the robot and payload. These forces are not only part of the feedback strategy in Equation (22), but also cause the system to deviate from its target configuration. Hogan and Flash described force commands from a human as a Cartesian stiffness control analogy[20]. If the human simply tries to hold the system stationary, variations in the systems configuration will cause the human to feel a reflection of the inertial and gravitational effects, which is the objective of the original control strategy. However, it is quite possible that these disturbance forces, while part of the environment, are not beneficial to the execution of the tasks 
and actually serve as a third avenue for the ship motion to disturb the performance of the system. As an example, Figure 46 through Figure 48 show the tip position of the system at sea state 5 when the operator is trying to hold the system stationary. Figure 45 shows the resulting interaction force between the payload and manipulator, measured by the force sensor. If the HAT control architecture is working properly, the operator will feel a scaled version of this force as illustrated in Figure 44. Our objective is to identify a control approach that can effectively mask off this ship motion disturbance force that is reflected back to the operator.

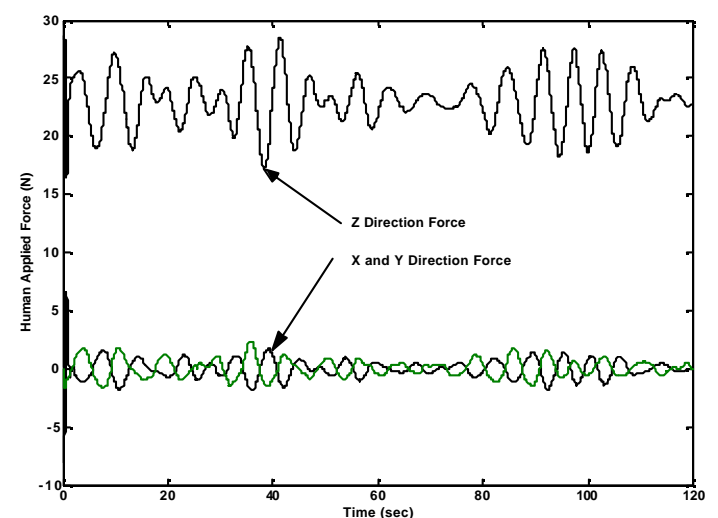

Figure 44: Human Forces at Sea State 5, No Compensation

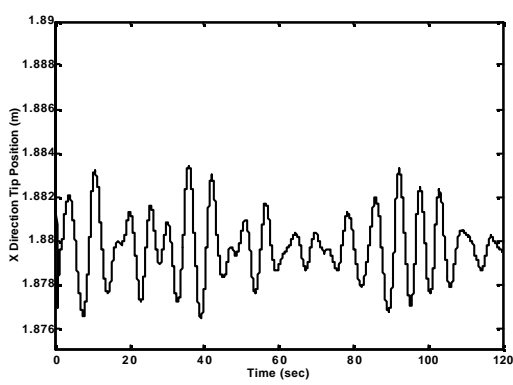

Figure 46: X-Position, No Compensation

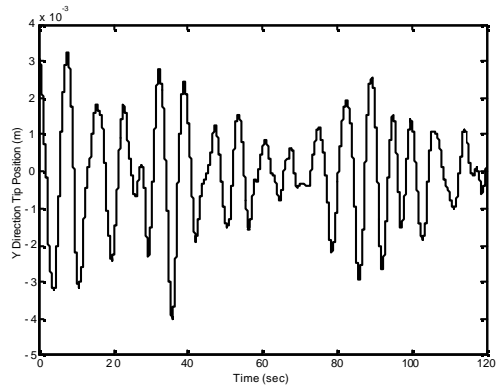

Figure 47: Y-Position, No Compensation

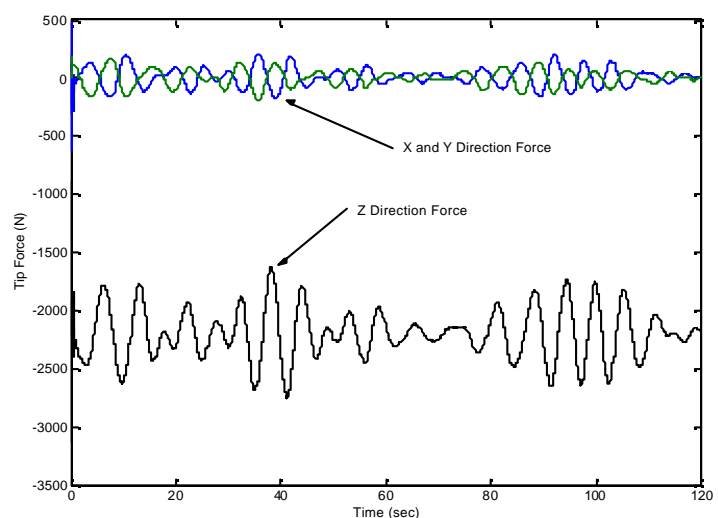

Figure 45: Tip Force at Sea State 5, No Compensation

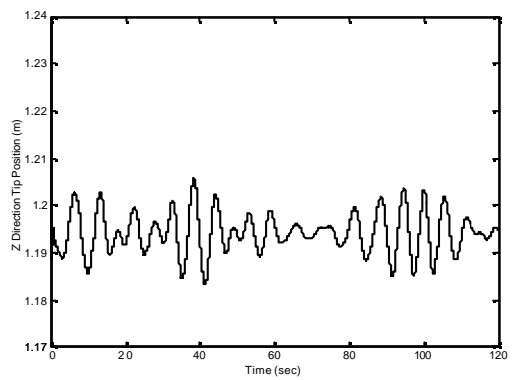

Figure 48: Z-Position, No Compensation

It is clear that, while attractive from the simplicity standpoint, the performance of the RLC will not sufficiently improve the fidelity of a ship-based HAT system. The advantage of the RLC approach is the lack of sensory feedback. If the period of the excitation is know and fixed, the RLC exploits the joint sensory feedback for mitigation of the periodic error signal. Unfortunately, while the sea state appears periodic, the frequency of the disturbance can shift by relatively large amounts from period to period. Attempts at adaptive RLC, varying the period of the RLC as the disturbance frequency varies, work only when the shift in disturbance frequency is slow. Subsequently, we must explore other avenues for mitigating the sea state disturbance. The basic problem is very similar to the work in noise cancellation. Widrow describes the feasibility of canceling noise in speech signals, specifically the problems with cockpit noise in flight. Engine noise contains strong periodic components, rich in harmonics, that occupy the same frequency band as the pilot's speech. These problems are very similar to the ship motion disturbance where the frequency and intensity are comparable to the command signals. For noise cancellation, a second microphone is placed at a suitable location in the cockpit to measure the ambient noise free of the pilot's speech. $\left[{ }^{21}\right]$ We adopt a similar strategy, called from here on the Ship Motion Compensation for Force 
Control Strategy (SMCFCS), for the reduction in ship motion disturbances on a force controlled manipulator. Instead of measuring background acoustical noise with a microphone, we measure background ship motion using accelerometers and inclinometers on the base of the manipulator. There are two slight modifications that must be considered. First, we are primarily interested in removing the disturbance force from the force sensor at the end of the robot. With acoustic noise cancellation, the noise measurement and speaker were both acoustical signals. For the force signal cancellation, the noise measurement is based on a displacement sensor (accelerometers and inclinometers) while the actual signal of interest is a force signal. Therefore, the algorithm has the additional constraint of transforming the noise measurement to a predicted force measurement. Second, the coordinate frame of the force transducer at the end of the robot is most likely in a different configuration than the accelerometers and inclinometers. Since the basic HAT control architecture is force based, it is straightforward to feedforward an effective sea state force to mask off the resulting inertial and gravitational loads. Note in Figure 31 that the force comparison between the human and payload is executed in the Cartesian frame. If we know the effective impedance of the payload, the disturbance force can be computed directly in the Cartesian frame if the accelerometers and inclinometers are calibrated with respect to this configuration on the robot's platform. The basic feedforward term for the noise cancellation is provided in Equation (23). This term represents the effective force due to the sea state, $\mathrm{F}_{\text {Seastate, }}$ defined with respect to the base of the robot. The first term represents the inertial forces due to the sea state while the second term accounts for the change in the gravitational field due to the ship configuration. The actual force error filtered through the accommodation matrix is expressed in Equation (24) where $\mathrm{R}_{\text {base }}^{\text {tip }}$ is simply the coordinate transformation for the base frame to the end-effector frame where the force signal, $\mathrm{F}_{\text {sensor }}$, is measured.

$$
\begin{aligned}
& F_{\text {SeaState }}=\left(M_{p}\left\{\begin{array}{l}
\ddot{x}_{\text {base }} \\
\ddot{y}_{\text {base }} \\
\ddot{z}_{\text {base }}
\end{array}\right\}-M_{p} g R_{\text {sea }}^{\text {base }}\left\{\begin{array}{l}
0 \\
0 \\
1
\end{array}\right\}\right) \\
& \Delta F=\alpha F_{\text {human }}-\left[\left(R_{\text {base }}^{\text {tip }}\right)^{t} F_{\text {sensor }}-F_{\text {SeaState }}\right]
\end{aligned}
$$

Figure 49 through Figure 53 display the response of the system with the addition of SMCFCS. Table 4 provides a comparison of the performance of the system, using the same operating conditions and sea state described in the previous simulations, under different levels of feedback. It is clear that this approach to noise cancellation looks promising. We must consider the fact that we assumed perfect knowledge of the payload mass. In addition, we did not account for any possible errors or dynamics in the sensing of the accelerations and inclination. 
Table 4: Control Performance Comparison

\begin{tabular}{|l|c|c|}
\hline Strategy & Force Variation $(\mathrm{N})$ & Position Variation $(\mathrm{mm})$ \\
\hline \hline No Compensation & $\Delta \mathrm{F}_{\mathrm{x}}=3.587$ & $\Delta \mathrm{x}^{\mathrm{tip}}=7.2$ \\
& $\Delta \mathrm{F}_{\mathrm{y}}=3.825$ & $\Delta \mathrm{y}^{\mathrm{tip}}=7.7$ \\
& $\Delta \mathrm{F}_{\mathrm{z}}=11.384$ & $\Delta \mathrm{z}^{\mathrm{ti}}=22.8$ \\
\hline SMCFCS w/Vertical Acceleration & $\Delta \mathrm{F}_{\mathrm{x}}=3.420$ & $\Delta \mathrm{x}^{\mathrm{tip}}=6.8$ \\
& $\Delta \mathrm{F}_{\mathrm{y}}=3.646$ & $\Delta \mathrm{y}^{\mathrm{tip}}=7.3$ \\
& $\Delta \mathrm{F}_{\mathrm{z}}=1.246$ & $\Delta \mathrm{z}^{\mathrm{tip}}=2.5$ \\
\hline SMCFCS w/X, Y, Z Acceleration & $\Delta \mathrm{F}_{\mathrm{x}}=2.366$ & $\Delta \mathrm{x}^{\mathrm{tip}}=2.5$ \\
& $\Delta \mathrm{F}_{\mathrm{y}}=2.102$ & $\Delta \mathrm{y}^{\mathrm{tip}}=4.2$ \\
SMCFCS w/X, Y, Z Acceleration and & $\Delta \mathrm{F}_{\mathrm{z}}=1.242$ & $\Delta \mathrm{z}^{\mathrm{tip}}=2.5$ \\
Inclination & $\Delta \mathrm{F}_{\mathrm{x}}=0.951$ & $\Delta \mathrm{x}^{\mathrm{tip}}=1.9$ \\
& $\Delta \mathrm{F}_{\mathrm{y}}=0.777$ & $\Delta \mathrm{y}^{\mathrm{tip}}=1.6$ \\
SMCFCS w/X, Y, Z Acceleration and & $\Delta \mathrm{F}_{\mathrm{z}}=1.217$ & $\Delta \mathrm{z}^{\mathrm{tip}}=2.4$ \\
Inclination and RLC & $\Delta \mathrm{F}_{\mathrm{x}}=0.598$ & $\Delta \mathrm{x}^{\mathrm{tip}}=1.2$ \\
& $\Delta \mathrm{F}_{\mathrm{y}}=0.774$ & $\Delta \mathrm{y}^{\mathrm{itp}}=1.5$ \\
& $\Delta \mathrm{F}_{\mathrm{z}}=0.847$ & $\Delta \mathrm{z}^{\mathrm{tip}}=1.7$ \\
\hline
\end{tabular}

It is clear that the SMCFCS with full acceleration and inclination feedback provides significant improvement in both the tracking (an increase in sensitivity by a factor of 9.5) and force reflection (reduction of disturbance force by a factor of 9.4). The fact that these two improvements are so similar should not be a surprise. As noted earlier, the motion of the tip is due to two stimuli: the disturbance force and the command. The SMCFCS masks off the disturbance force in the HAT algorithm that generates motion commands to the manipulator. So, the SMCFCS eliminates the disturbance from the command that significantly reduces the relative motion between the ship and the operator. The SMCFCS framework is based in the inertial frame of the robot (the same frame as the HAT methodology), instead of the joint space. This dramatically simplifies the complexity of the algorithm. It may be possible to further reduce the sensitivity of the system to the sea state. However, the disturbance forces and tracking performance are bordering on human sensitivity. Any additional gain would not be perceived by the operator. The only motivation would be operation at sea states 6 and higher.

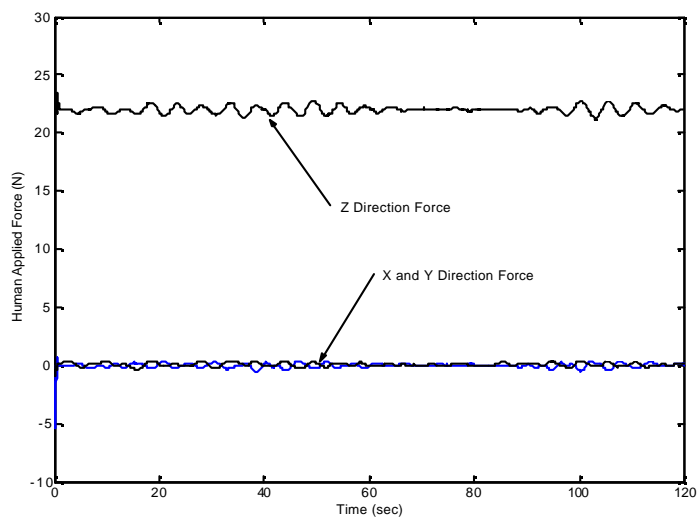

Figure 49: Human Forces at Sea State 5, SMCFCS

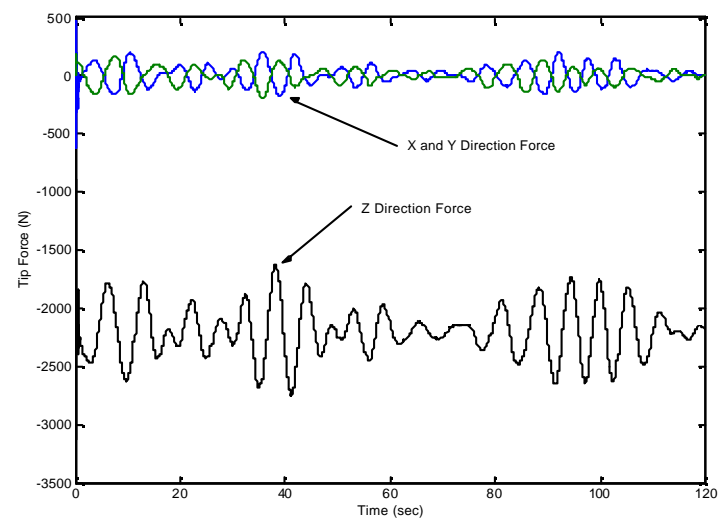

Figure 50: Tip Force at Sea State 5, SMCFCS 


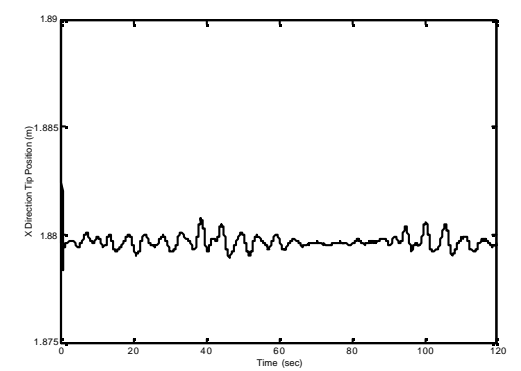

Figure 51: X-Position, SMCFCS

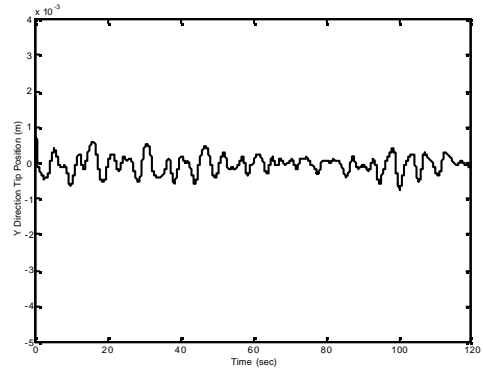

Figure 52: Y-Position, SMCFCS

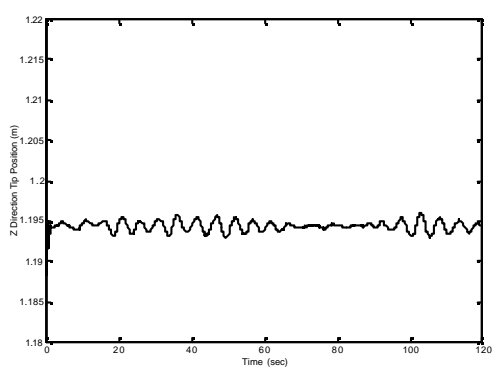

Figure 53: Z-Position, SMCFCS

\section{Conclusions ANd Results}

This report has described the fundamental problems associated with motion control of manipulation systems operating on the deck of a moving ship. We provided a brief survey of present wave modeling techniques and ship motion simulation procedures. This is followed by a methodology to compute the dynamic equations of motion, using energy methods, of a general serial link manipulator on a six-degree of freedom base. We provide as an example a three-degree of freedom manipulator and show, through simulation, the impact of wave generated disturbance on the tracking control of this system. As an example system, we developed a detailed model of an ORNL HAT system to demonstrate the impact sea states have, through simulation, on a typical human amplification technology system operating on the deck of a ship experiencing heavy sea states. We first demonstrated human amplification through simulation showing comparable performance as that experienced on the real system. These simulation studies included force tracking, strength amplification, and contact stability. This was followed by a series of simulations that displayed the impact ship motion has on strength amplification on a moving platform. The test case was a strength amplification factor of 100:1 with a $2224 \mathrm{~N}$ payload. The gravitational load experienced by the operator is $22.2 \mathrm{~N}$ (5 lbs). At sea state 5, the variation in the vertical load was $11.4 \mathrm{~N}, 51 \%$ of the load. This variation in the load generated a disturbance motion of $22.8 \mathrm{~mm}$ in the vertical direction, dramatically limiting the positioning resolution of the arm during operation. Our initial control investigation focused on Repetitive Learning Control. This control methodology is based on the elimination of periodic disturbances through a simple feedforward strategy. The advantage of this approach is its simplicity. If the disturbance period is known and of a fixed period, there is no need for any additional sensory feedback. If the disturbance is periodic, but of an unknown frequency, we can use on-line frequency identification techniques to extract the period of the disturbance. Simulation results demonstrated the feasibility and resulting performance. However, realistic sea state disturbances, while low in frequency, are not purely periodic. All of our attempts at frequency adaptation for the Repetitive Learning Control provided negligible improvement in the HAT performance when we introduced realistic sea state disturbances to the system.

Our second approach to masking off the sea state focused on exploiting the force control methodology. We assume that the HAT system has a stationary base from which it operates. This base can be a mobile platform, but during sensitive HAT operations, we assume the base is stationary. The fundamental problem that we addressed is that the sea state generated accelerations and shifts in the direction of the gravitational field on the system and payload. The HAT control algorithm is based on sensory feedback of the human applied force and the force, measured at the interface between the manipulator and payload, experienced by the payload. This end-effector force signal, which is fed back to the HAT controller, includes all of the 
disturbance forces (acceleration and gravitational) due to the sea state. We assume that the mass of the payload is known or can be rapidly measured during an initial pick up of the payload. We also assume that we can measure the acceleration and inclination of the base of the robot using conventional accelerometers and inclinometers. We believe these assumptions are valid due to the low frequency nature of the disturbances. We exploit the fact that the HAT control methodology is based on a task space formulation (the forces of the human and payload are defined with respect to the Cartesian coordinate frame of the robot). We then use the sensory feedback of the base inclination and acceleration to formulate the disturbance force, due to the sea state, experienced by the HAT system. The control strategy then consists of feeding forward this disturbance to mask off its effect on the HAT system. Simulation results illustrate a significant reduction in the disturbance experienced by the operation. Under the same operating conditions, the maximum vertical disturbance force was reduced from $11.4 \mathrm{~N}$ to $0.847 \mathrm{~N}$. In addition, the tip position error reduced from $22.8 \mathrm{~mm}$ to $1.7 \mathrm{~mm}$.

There were two fundamental contributions of the research. The first was the development of a new methodology to rapidly compute the full nonlinear dynamic equations of motion of a general manipulation system experiencing the full six degrees of disturbances due to a sea state. We demonstrated how this methodology could be easily included in a numerical simulation tool such as Simulink. Such a tool is extremely valuable in terms of exploring design and control issues for maritime systems. The second contribution was the development of a new control algorithm for compensation of sea state disturbances on a maritime Human Amplification System. Simulation studies showed an order of magnitude improvement in both the tracking performance and reduction in the disturbance force experienced by the operator. Future efforts are focusing on the experimentally validating this control methodology. These experiments will be based on a large sea state simulator that will induce the inclination and acceleration loads on our ORNL IRAD HAT system. 


\section{REFERENCES}

[1] T. Deeter, G. Koury, K. Rabideau, M. Leahy, and T. Turner, "The Next Generation Munitions Handler Advanced Technology Demonstrator Program," Proceedings of the 1997 IEEE International Conference on Robotics and Automation, pp. 341-345, 1997.

[2] R. J. Henry, Z. N. Masoud, A. H. Nayfeh, and D. T. Mook, "Cargo Pendulation Reduction on ShipMounted Cranes Via Boom-Luff Angle Actuation," submitted for publication, Journal of Vibration and Control.

[3] C-M. Chin, A. H. Nayfeh, and D. T. Mook, "'Dynamics and Control of Ship-Mounted Cranes," AIAA Paper No. 98-1731, 39 ${ }^{\text {th }}$ AIAA/ASME/ASCE/AHS/ASC Structures, Structural Dynamics, and Materials Conference, Long Beach, CA, April 1998.

[4] W. Lacarbonara, R. R. Soper, A. H. Nayfeh, and D. T. Mook, “'A Fully Passive Architecture for Pay-Load Pendulation Control in Cranes," International Conference on Monitoring and Control of Marine and Harbour Structures, Genoa, Italy, 1999.

[5] B. Kimiaghalam, A. Homaifar, and M. Bikdash, "Feedback and feedforward control law for a ship crane with the Maryland rigging system", Proceedings of the 2000 ACC.

[6] http://www.ait.nrl.navy.mil/people/dmaxwell/mocove.html

[7] http://www.lakehurst.navy.mil/lke-bdo/motionpl.htm

[8] http://www.arl.psu.edu/facilities/facilities/warminster/warminster.html

[9] R. Bhattacharyya, Dynamics of Marine Vehicles, John Wiley \& Sons, 1978.

[10] A. Engle, A. Lin, N. Salvensen, and Y. Shin, "Application of 3-D Nonlinear Wave-Load and Structural Response Simulations in Naval Ship Design," Naval Engineers Journal, Vol. 109, No.3, May 1997.

[11] M. Abkowitz, "Applications of the Spectral Techniques to Design and Operation," Proceedings of the Society of Naval Architects and Marine Engineers Sea Keeping Symposium, 1973.

[12] T. Lamb, "Organization Theory and Shipbuilding -- A Brief Overview," Marine Technology, April 1992.

[13] N. Salvensen, O. Tuck, and O. Faltinsen, "Ship Motions and Sea Loads," Transactions of the Society of Naval Architects and Marine Engineers, Vol.78, pp.250-287, 1970.

[14] W. Price, and R. Bishop, Probabilistic Theory of Ship Dynamics, Halsted Press, 1974.

[15] W. Price, Dynamics of Ships, Scholium International, 1991.

[16] T. Ogilvie, "Fundamental Assumptions in Ship-motion Theory," The Dynamics of Marine Vehicles and Structures in Waves, Edited by R.E.D. Bishop and W.G. Price, Institute of Mechanical Engineers, London, 1975, pp.135-145.

[17] Sadegh, N., Horowitz, R., Kao, W, and Tomizuka, M., "A Unified Approach to the Design of Adaptive and Repetitive Controllers for Robotic Manipulators," ASME's Journal of Dynamic Systems, Measurement and Control, Vol. 112, pp.618-629, Dec. 1990.

[18]Shaw, f., and Srinivasan, K., "Discrete-Time Repetitive Control System Design Using the Regenerative Spectrum," ASME's Journal of Dynamic Systems, Measurement, and Control, Vol. 115, pp.228-237, June 1993.

[19] Whitney, D., "Historical Perspective and State of the Art in Robot Force Control," International Journal of Robotics Research, Vol. 6, No. 1, pp. 3-14, 1987.

[20] Flash, T, and Hogan, N., "The coordination of Arm Movements: An experimentally Confirmed 
Mathematical Model, Journal of Neuroscience, Vol 5, pp. 1688-1703.

[21] Widrow, B., and Stearns, S., Adaptive Signal Processing, Prentice-Hall, Inc., Englewood Cliffs, NJ, 1985, pp.337-339. 


\section{Appendix A: Symbolic Computation of 1 DOF System, 3 Sea States}

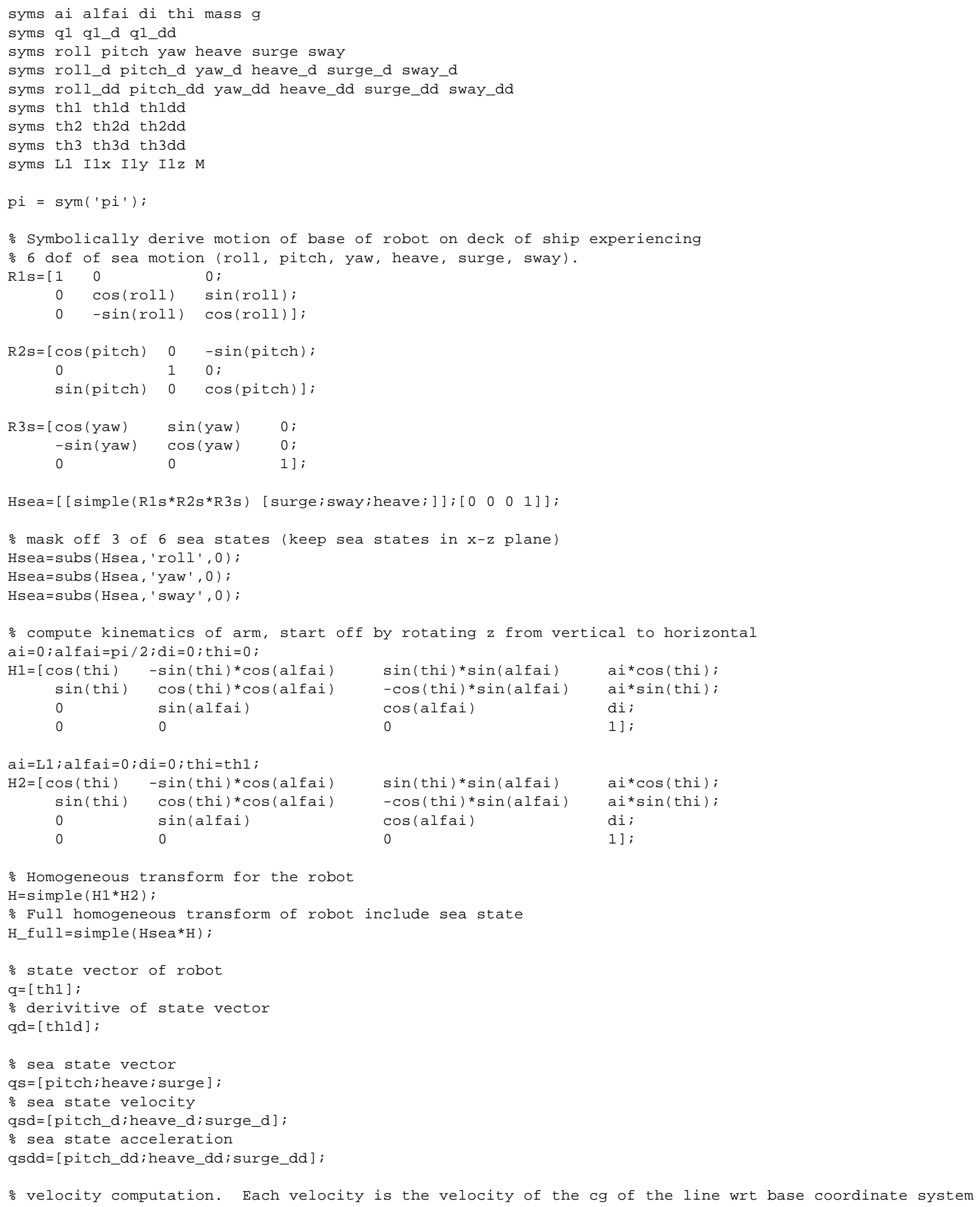




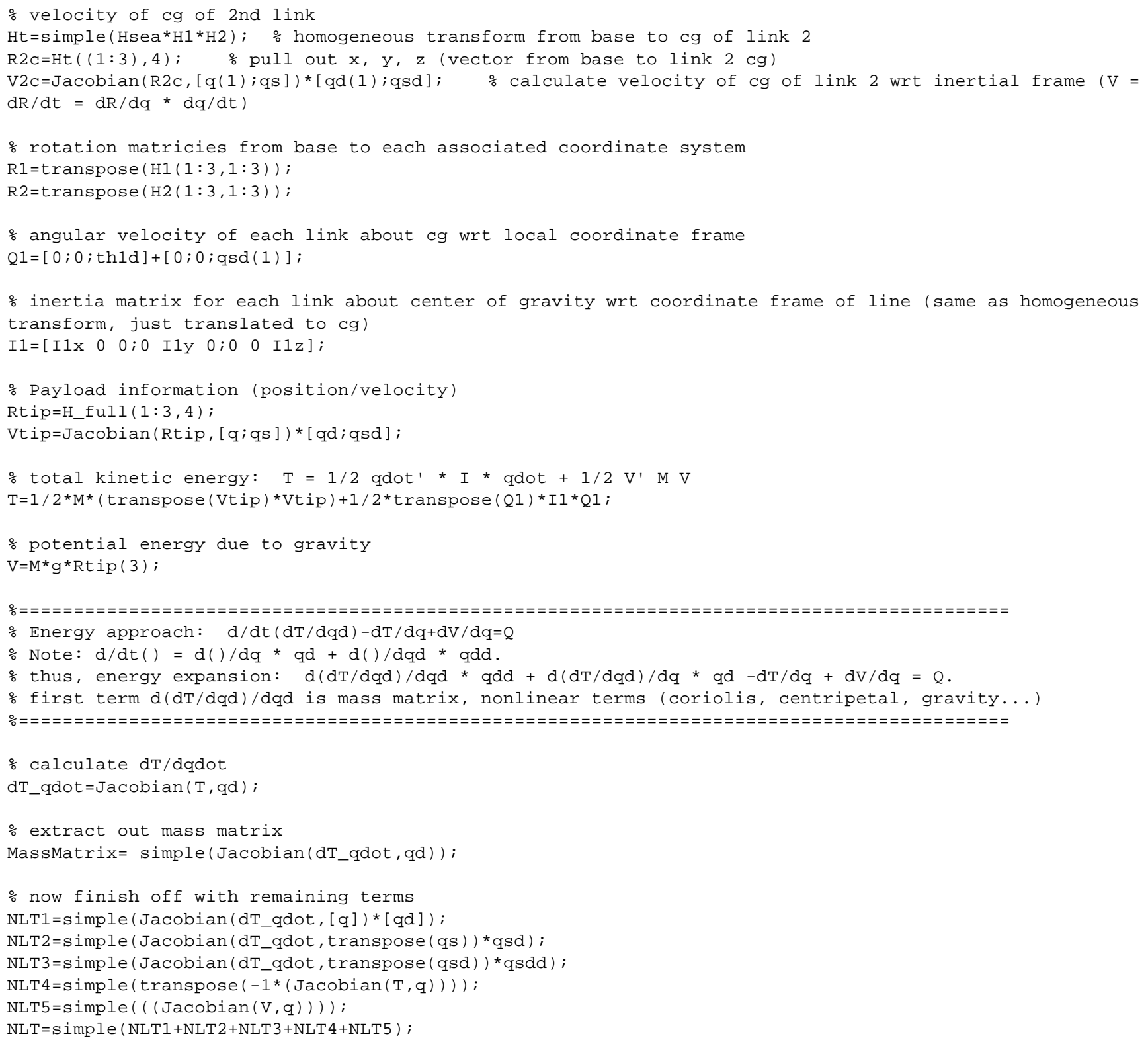

Appendix A-2 


\section{Appendix B: Symbolic Computation of 3 DOF System, Full Sea State}

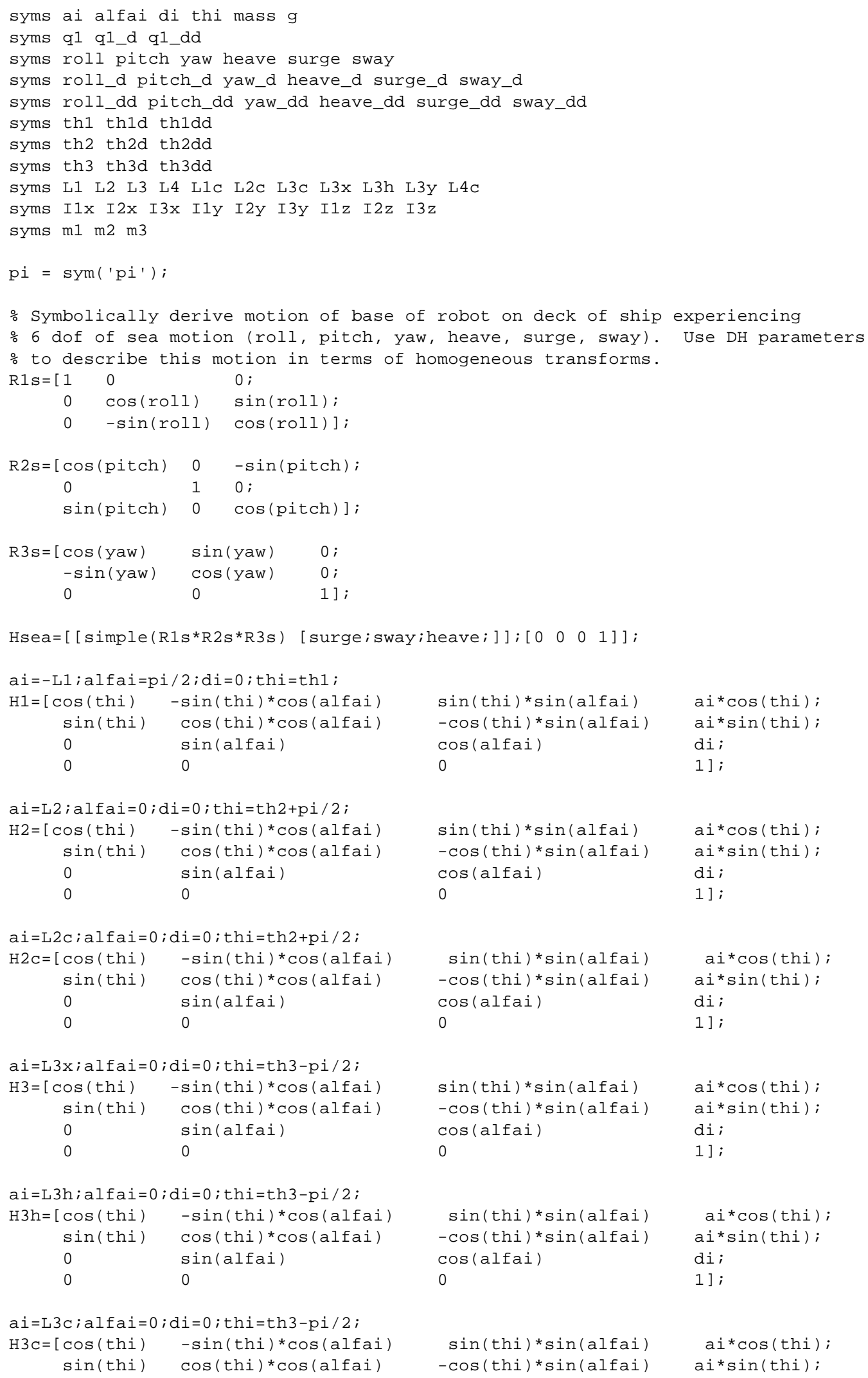

Appendix B-1 


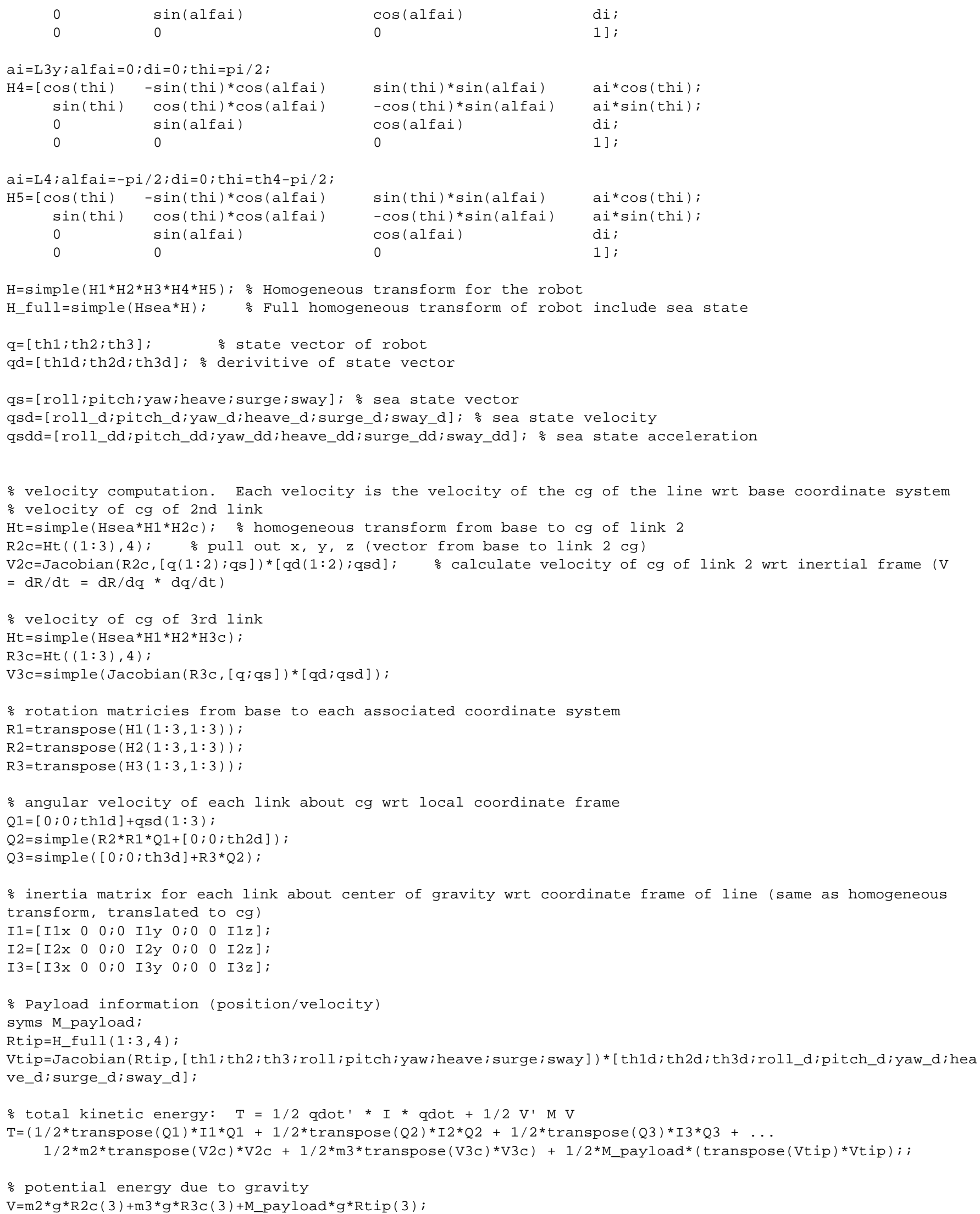

Appendix B-2 
\% calculate dT/dqdot

dT_qdot=Jacobian $(T, q d)$;

o extract out mass matrix

MassMatrix= simple (Jacobian (dT_qdot, qd)) ;

\% now finish off with remaining terms

NLT1=simple (Jacobian (dT_qdot, $[q])$ * [qd] ) ;

NLT2=simple (Jacobian (dT_qdot, transpose (qs) )*qsd);

NLT3=simple (Jacobian (dT_qdot, transpose (qsd)) *qsdd);

NLT 4=simple $(-1 * \operatorname{transpose}((\operatorname{Jacobian}(T, q))))$;

NLT5=simple $(((\operatorname{Jacobian}(V, q))))$;

\% translate to $\mathrm{C}$-code

MassMatrix_cC=Ccode (MassMatrix);

NLT1 $\mathrm{CC}=\mathrm{CCOde}(\mathrm{NLT} 1)$;

NLT2_CC=CCOde (NLT2);

NLT3 $\mathrm{CC}=\mathrm{ccode}(\mathrm{NLT} 3)$;

NLT 4_CC=CCOde (NLT4);

NLT5 cC=ccode (NLT5);

o calculation of jacobian from tip frame to joint space

LDRDJacobian=simple (Jacobian $(H(1: 3,4),[$ th 1 ; th2; th 3$]))$;

LDRDJacobian_CC=CCode (LDRDJacobian) ;

Appendix B-3 


\section{Appendix C: RLC Sensitivity Analysis}

There are a great many tools available for the synthesis and analysis of linear control systems. In the following sections, we explore the frequency response representation of a basic repetitive control algorithm. This analysis will provide insight into why a repetitive control algorithm can improve the tracking performance of simple linear controller for harmonic reference signals, while at the same time reject disturbance signals of the same frequency at the same time.

\section{Simplified Model}

Our initial intention was to explore the sensitivity of repetitive control methodologies to variations in the disturbance frequency. Specifically, how much variation in the disturbance frequency is allowable before the repetitive control either provide no benefit or even degrades performance. As an example, we explore the simplest model, a first order plant and controller with a series repetitive control, shown in Figure 1. This is actually representative of a wide array of controlled plants operating in the vicinity of their crossover frequency.

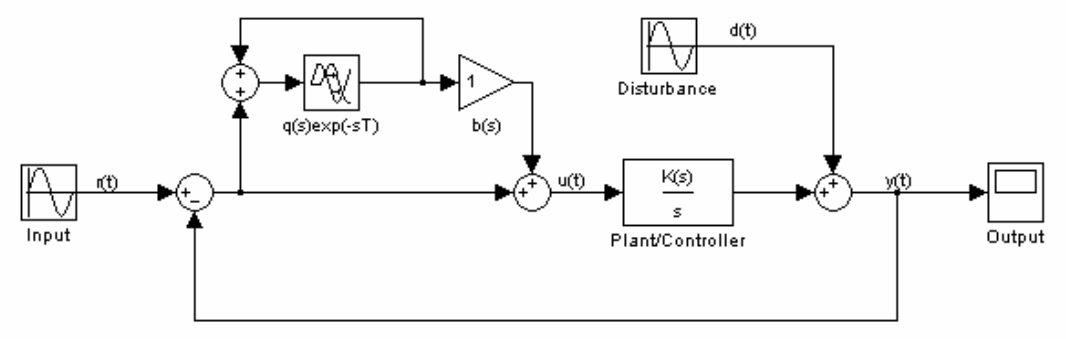

Figure 54: Simplified Plant Model

To begin, we look at the frequency response of the reduced repetitive control element. Equation (25) shows the basic transfer function representation of the repetitive control element in Figure 54.

$$
G_{r c}(j \omega)=\frac{1}{1-e^{-j \omega T}}
$$

At $\omega=2 \pi / T, G_{\mathrm{rc}}$ approaches infinity. Essentially, the repetitive control is providing infinite gain at the target frequency without numerically requiring high gains. Unlike conventional approaches to high gain feedback, the use of the repetitive control provides the benefits of high gain feedback, but only at a specific frequency without sacrificing stability.

There are a number of methods to approximate delay type functions. Beghi (1997) provides insight into alternative methods for approximating delay elements in feedback. We now focus on a similar approach for approximating the transfer function of the RC algorithm outlined above. Equation (25) can be reordered to provide an input output relationship between the error signal, $\mathrm{e}(\mathrm{t})$, going into the element and the modified error signal coming out of the RC element.

$$
\tilde{\mathrm{e}}(\mathrm{t})=\mathrm{e}(\mathrm{t})+\tilde{\mathrm{e}}(\mathrm{t}-\mathrm{T})
$$

Figure 41 shows the resulting waveform of Equation (26) when assuming e(t) is a unit step function. It is possible to decompose Figure 41 into three separate elements: a ramp, a step, and a sawtooth waveform. Figure 42 shows the resulting components. 


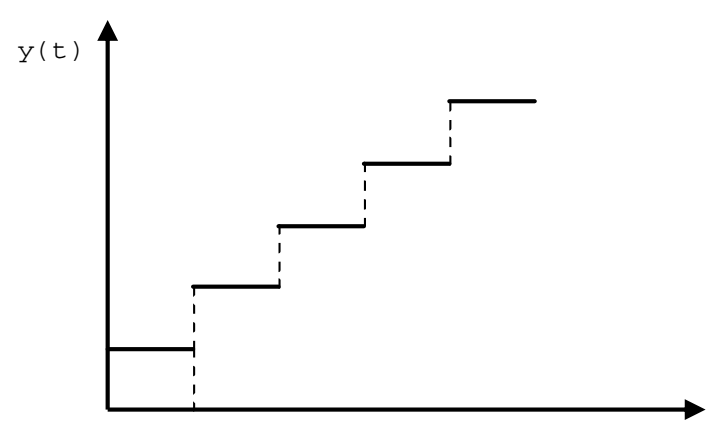

Figure 55: Step Response of RLC Element

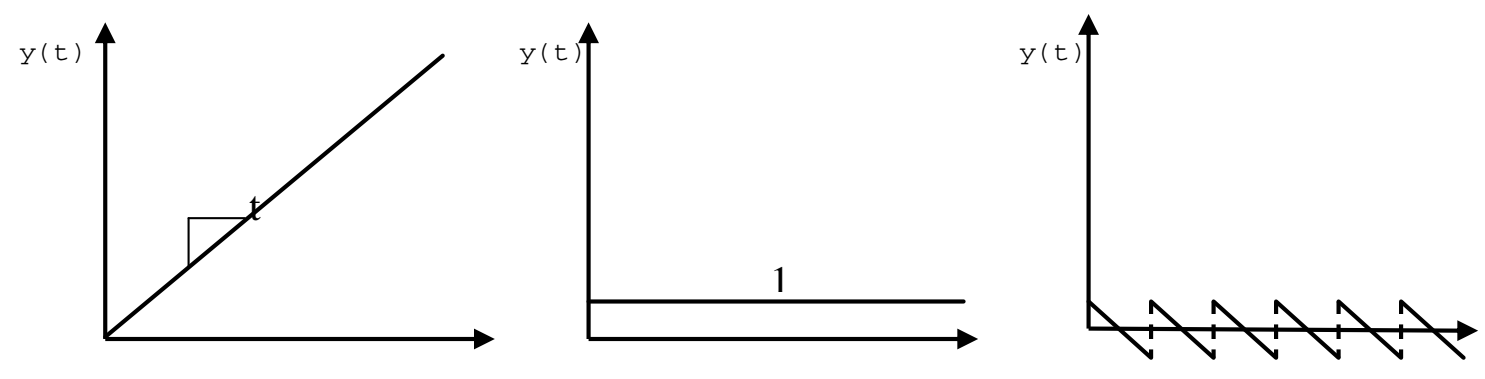

Figure 56: Components of overall response

By expanding the waveform in Figure 55 to the three elements, it becomes trivial to identify by inspection the resulting time response equation assuming the input to the system is a step input $\mathrm{u}(\mathrm{t})$. 


$$
\tilde{\mathrm{e}}(\mathrm{t})=\frac{\mathrm{t}}{\mathrm{T}}+\frac{1}{2}+\frac{1}{\pi} \sum_{\mathrm{i}=1}^{\mathrm{N}} \frac{1}{\mathrm{i}} \sin ^{2}\left(\frac{2 \pi \mathrm{i}}{\mathrm{T}} \mathrm{t}\right)
$$

We can then easily transform to the frequency domain.

$$
\tilde{\mathrm{E}}(\mathrm{s})=\frac{1}{\mathrm{Ts}^{2}}+\frac{1}{2 \mathrm{~s}}+\frac{2}{\mathrm{~T}} \sum_{\mathrm{i}=1}^{\infty} \frac{1}{\mathrm{~s}^{2}+\left(\frac{2 \pi \mathrm{i}}{\mathrm{T}}\right)^{2}}
$$

Therefore, we can express the resulting transfer function of Equation (28) by removing the step component (1/s) from Equation (27).

$$
\mathrm{G}_{\mathrm{rc}}(\mathrm{s})=\frac{1}{\mathrm{Ts}}+\frac{1}{2}+\frac{2}{\mathrm{~T}} \sum_{\mathrm{i}=1}^{\mathrm{N}} \frac{\mathrm{s}}{\mathrm{s}^{2}+\left(\frac{2 \pi \mathrm{i}}{\mathrm{T}}\right)^{2}}
$$

We can now combine terms in an effort to have an expression that provides the resulting poles and zeros of the system.

$$
\mathrm{G}_{\mathrm{rc}}(\mathrm{s})=\frac{(2+\mathrm{Ts}) \prod_{\mathrm{i}=1}^{\mathrm{N}}\left(\mathrm{s}^{2}+\left(\frac{2 \pi \mathrm{i}}{\mathrm{T}}\right)^{2}\right)+4 \mathrm{~s}^{2} \sum_{\mathrm{i}=1}^{\mathrm{N}} \prod_{\substack{\mathrm{j}=1 \\ \mathrm{j} \neq \mathrm{i}}}^{\mathrm{N}}\left(\mathrm{s}^{2}+\left(\frac{2 \pi \mathrm{j}}{\mathrm{T}}\right)^{2}\right)}{2 \mathrm{Ts} \prod_{\mathrm{i}=1}^{\mathrm{N}}\left(\mathrm{s}^{2}+\left(\frac{2 \pi \mathrm{i}}{\mathrm{T}}\right)^{2}\right)}
$$

First, Figure 57 displays the resulting step response of the $\mathrm{G}_{\mathrm{rc}}(\mathrm{s})$ for $\mathrm{N}=2,5,10$, and 20.
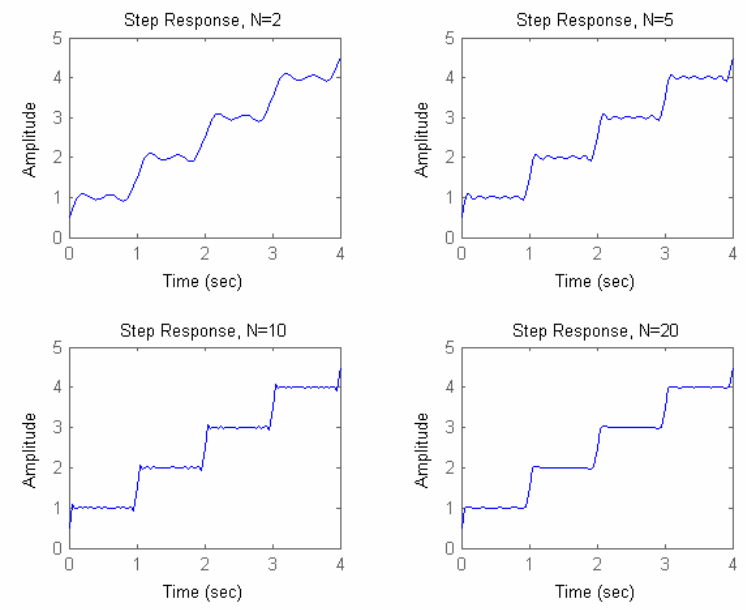

Figure 57: Comparison of step response

Figure 57, assuming $\mathrm{T}=1 \mathrm{sec}$, shows that there is good agreement between the theoretical step response in Figure 55 and the step response due to the approximation. It is clear from the model in Equation (30) that the system's poles consist of an infinite set of complex conjugate pair spaced on the $j \omega$-axis at $\omega=2 \pi \mathrm{i} / \mathrm{T}$. Unfortunately, it is not as straightforward to extract the zeros of the system. Figure 58 shows the pole-zero map of $\mathrm{G}_{\mathrm{rc}}(\mathrm{s})$ as a function of variations in $\mathrm{N}$ as before. 

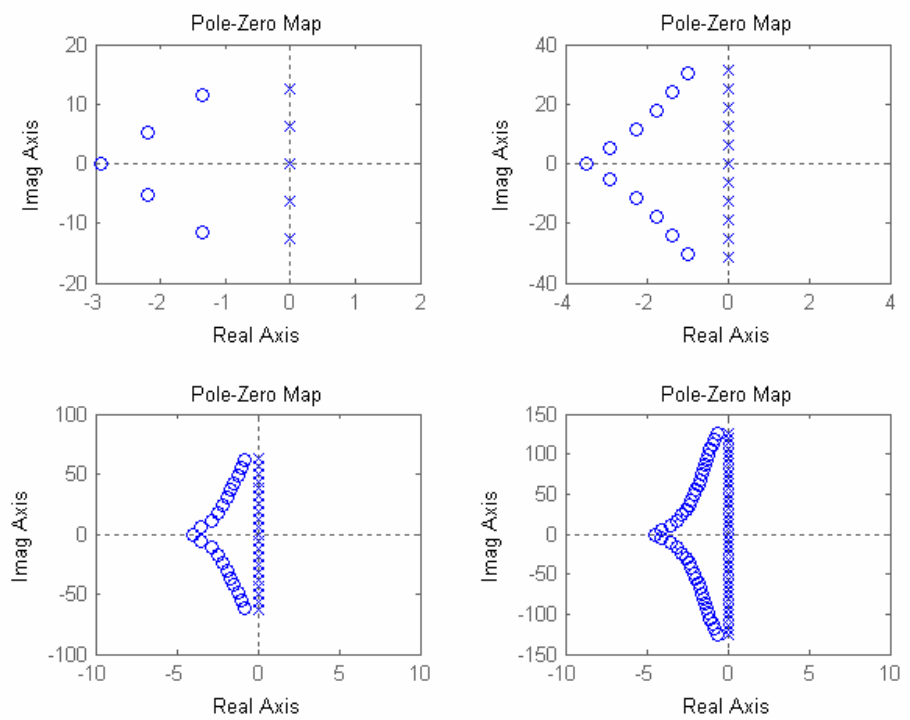

Figure 58: Pole-Zero map of $\mathrm{G}_{\mathrm{rc}}$

It is clear that for every pair of poles on the $\mathrm{j} \omega$-axis, there is a complementary complex conjugate pair zeros in the left half plane. Increasing the model order retains the lower order pole-zero pairs. Additional information can be gained by investigating the system's frequency response. Figure 58 and Figure 59 show the frequency response of three variations of Equation (25). The first display in both figures is the exact magnitude and phase of the transfer function. Many times, it is advantageous to have an approximate model representing the delay, possible as a series of poles and zeros. The second display in both figures shows Equation (25) with an $8^{\text {th }}$ order Pade approximation of $\mathrm{e}^{\mathrm{sT}}$. Clearly, the Pade approximation provides an excellent representation of the system for the first two pairs of complex poles and zeros, but the system rapidly degenerates. The third displays in both figures shows the same system with an $8^{\text {th }}$ order Fourier approximation of the delay derived in Equation (30). It is clear that, with the same order model as used for the Pade approximation, the Fourier model faithfully reproduces the first four modes without any distortion of the higher modes as is evident in the Pade approximation. The importance of this representation becomes clear when attempting to utilize conventional control synthesis procedures and bound stability margins for the final system. 


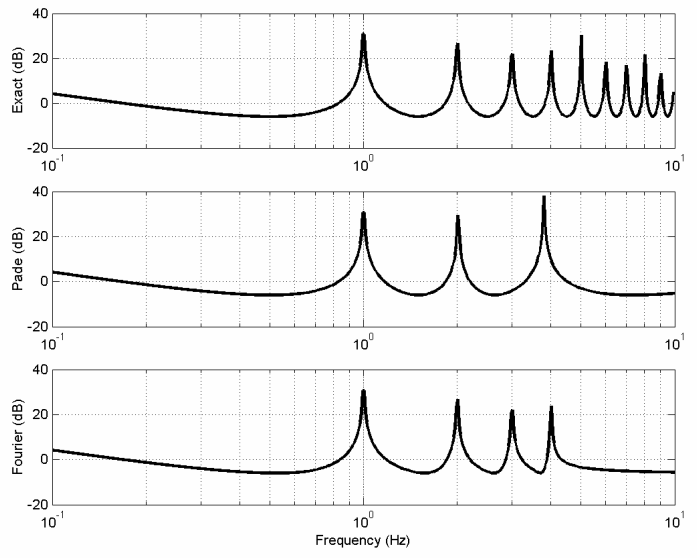

Figure 59: Comparison of Magnitude Response
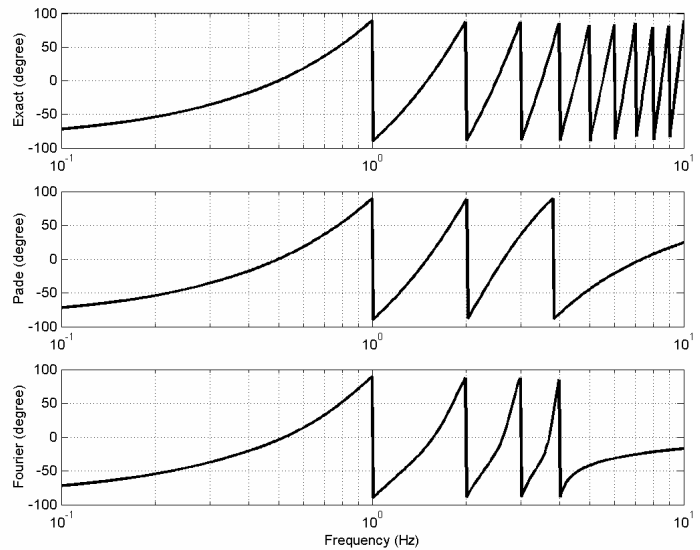

Figure 60: Comparison of Phase Response

\section{System Performance}

We now begin exploring the tracking performance and disturbance rejection capacity of a repetitive control strategy. We focus on implementation of simplified first and second order plants. Specifically, we will quantify the sensitivity with respect to variations in excitation frequency. Equation (31) describes the output as a function of the disturbance, $\mathrm{d}(\mathrm{t})$, and input, $\mathrm{r}(\mathrm{t})$ in terms of the Laplace variable, $\mathrm{s}$ and period of disturbance, $\mathrm{T}$.

$$
\mathrm{Y}(\mathrm{s})=\frac{\mathrm{K}}{\mathrm{s}\left(1-\mathrm{e}^{-\mathrm{sT}}\right)+\mathrm{K}} \mathrm{R}(\mathrm{s})+\frac{\mathrm{s}\left(1-\mathrm{e}^{-\mathrm{sT}}\right)}{\mathrm{s}\left(1-\mathrm{e}^{-\mathrm{sT}}\right)+\mathrm{K}} \mathrm{D}(\mathrm{s})
$$

We will begin by analyzing the disturbance response of the plant. Equation (32) expresses the frequency response of $Y(s)$ as a function of the disturbance $D(s)$ after substitution of $s=j \omega$ and $e^{-s T}=\cos (\omega T)-j$ $\sin (\omega \mathrm{T})$, and some minor algebraic manipulation.

$$
\frac{Y(j \omega)}{D(j \omega)}=\frac{2 \omega^{2}(1-\cos (\omega \mathrm{T}))-\omega \mathrm{K} \sin (\omega \mathrm{T})+j \omega \mathrm{K}(1-\cos (\omega \mathrm{T}))}{(\mathrm{K}-\omega \sin (\omega \mathrm{T}))^{2}+\omega^{2}(1-\cos (\omega \mathrm{T}))^{2}}
$$

Figure 60 shows the resulting magnitude of Equation (32) over a small frequency range around the disturbance period. For this example, $\mathrm{T}=4 \mathrm{sec}$, and $\mathrm{K}=6.3095$ and the frequency varies from 0.1 to 10 $\mathrm{Hz}$. 


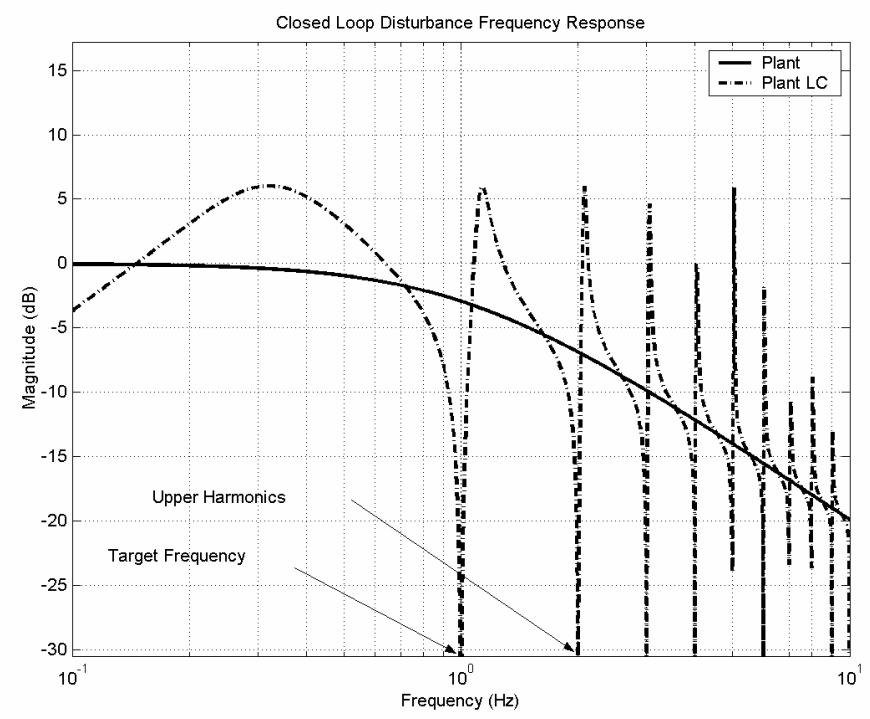

Figure 61: Disturbance Response

The figure clearly demonstrates the desired result, an output with zero amplitude at the disturbance frequency. There is also the corresponding zeros at the higher harmonics. However, a deviation of only $12 \%$ on either side of the target frequency results in a disturbance response magnitude that is actually greater than what would be present without the repetitive control, thus highlighting the sensitivity issue. Equation (33) expresses the frequency response of $Y(j \omega)$ as a function of the input $R(j \omega)$, derived from the system in Figure 54.

$$
\frac{Y(j \omega)}{R(j \omega)}=\frac{K(K-\omega \sin (\omega T))-j \omega K(1-\cos (\omega T))}{(K-\omega \sin (\omega T))^{2}+\omega^{2}(1+\omega \cos (\omega T))^{2}}
$$

Figure 62 shows the corresponding magnitude over a small frequency range around the disturbance period. For this example, $\mathrm{T}=1 \mathrm{sec}$, and $\mathrm{K}=6.3095$ and the frequency varies from 0.1 to $10 \mathrm{~Hz}$.

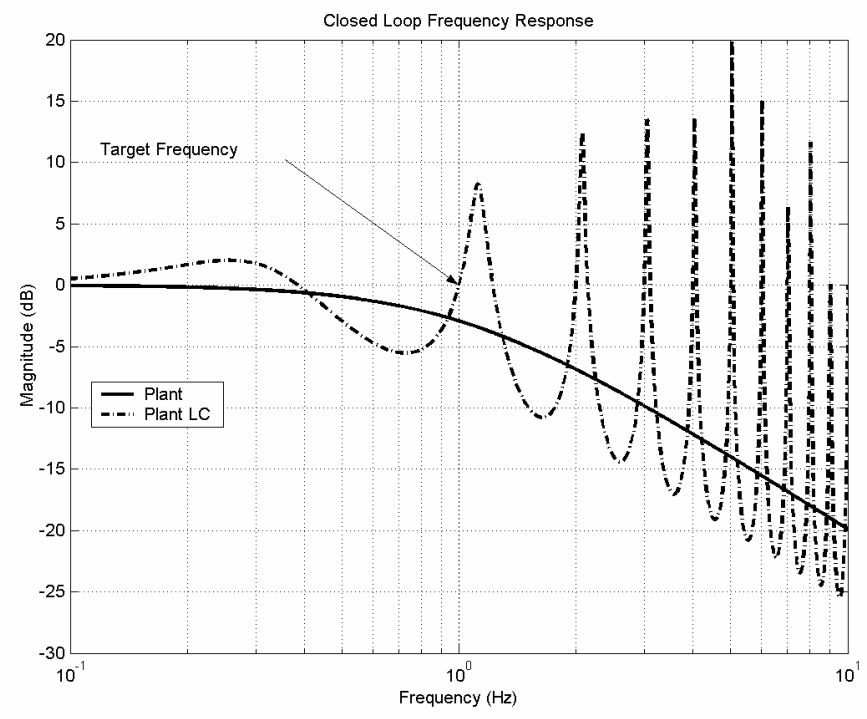

Figure 62: Closed Loop Tracking Performance

Figure 61 and Figure 62 emphasize two important characteristics of RLC. First, at the target frequency, Appendix C-6 
the closed loop system has perfect tracking performance while simultaneously rejecting a disturbance of the same frequency. Second, the control designer is faced with the sensitivity of the tracking and disturbance response with respect to both variations in the target frequency as well as higher order harmonics. As Figure 61 and Figure 62 illustrate, the system degenerates when the excitation or disturbance frequency deviates slightly from this target frequency. It is possible to resolve the distortion of the closed loop transfer function, at the lower frequencies, by applying a complimentary prefilter. However, we are limited to the first and seconds mode. To operate beyond this would require an extremely high ordered filter to generate the large amplitude swings over very small frequency ranges.

As an example of the time domain response, we use the simulink model in Figure 54 with both the reference, $\mathrm{r}(\mathrm{t})$ and disturbance, $\mathrm{d}(\mathrm{t})$ having the same frequency $(1 \mathrm{~Hz})$. The amplitude of the reference signal is 1 while the disturbance has an amplitude of 2. There is a 72 degree phase (selected randomly) difference between the reference and disturbance signals. The challenge with this example is that the desired trajectory has the same frequency as the disturbance. Traditional loopshaping control approaches assume the disturbance frequencies are higher than the desired operational bandwidth of the system. Subsequently, the controller is designed for high gain feedback in the operational frequency range and low gains in the frequency range of the disturbance. The distinct advantage of $\mathrm{RC}$ algorithms is that they provide excellent disturbance rejection in the operational bandwidth of the system.
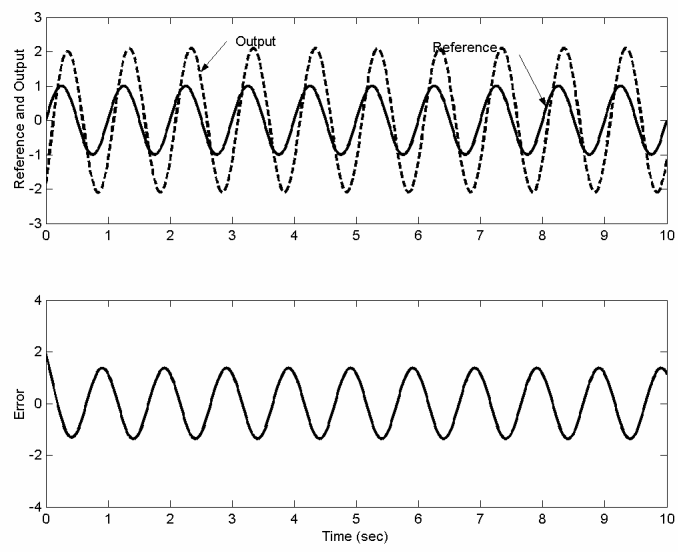

Figure 63: No RLC
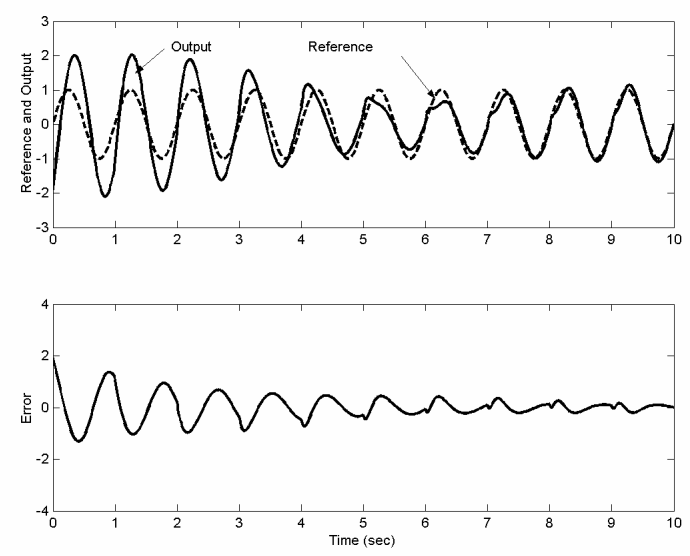

Figure 64: With RLC

In the above example, the reference input was sinusoidal and was of the same frequency as the disturbance. Figure 63 shows the system tracking without RLC while Figure 64 shows the same system with RLC. Clearly, the RLC provides excellent tracking of a periodic signal even if the disturbance has the same frequency as reference signal. We now follow this with the more conventional step response. The first display, Figure 64, is of a step response at the moment that learning begins. As before, the magnitude of the disturbance is twice the magnitude of the reference command. The majority of the initial error is due to the disturbance. To illustrate this, the command is delayed for 30 seconds before the step to provide ample time for the learning to converge. The response is displayed in Figure 66. 

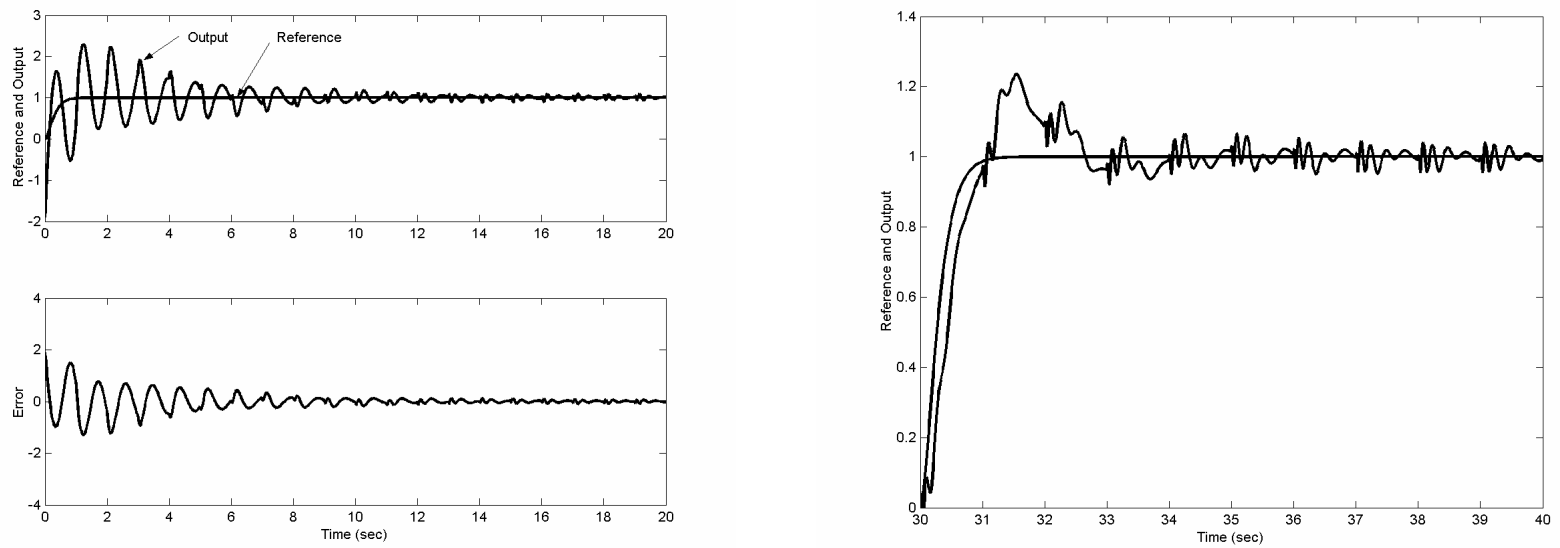

Figure 65: Step Response

Figure 66: Step Response after Delay

The forgoing analysis demonstrates the impressive performance of Repetitive Control algorithms when tracking sinusoidal commands and/or rejecting sinusoidal disturbances of fixed frequency. While many control methodologies focus on tracking performance and disturbance rejection, Repetitive Control has the distinct advantage of providing disturbance rejection of signals that fall within the operational frequency range of the controlled system. It is thus possible to design a system to operate beyond the frequency range of the disturbance, for that matter even track commands of the same frequency. The motivation for this paper was to provide a frequency domain explanation of the cost and benefit of RC. We have provided a new modeling approach for the RC that enables easy synthesis with conventional linear control methodologies. However, the analysis clearly illustrates that the addition of the Repetitive Control algorithm does distort the closed loop frequency response above and below the target frequency. Subsequent work will demonstrate simple loopshaping methods that focus on the design of prefilters to the system that correct for the frequency warping. In addition, there is a need for either increasing the robustness of the algorithm to variations or errors in the target frequency or providing some form of adaptation. 
ORNL/TM-2003/233

\section{INTERNAL DISTRIBUTION}

1. E. C. Fox

2-4. J. F. Jansen

5-7. L. J. Love

8-10. F. G. Pin

11. M. Noakes

12. P.D. Lloyd

13. Central Research Library

14. ORNL Laboratory Records BRC

15. ORNL Laboratory Records BOST

\section{EXTERNAL DISTRIBUTION}

16. Dr. Behzad Kamgar-Parsi, Autonomous and Intelligent Systems, Office of Naval Research, 800 North Quincy Street, Arlington, VA 22217. 